\title{
Combined shape and topology optimization for minimization of maximal von Mises stress
}

Lian, Haojie; Christiansen, Asger Nyman; Tortorelli, Daniel A.; Sigmund, Ole; Aage, Niels

Published in:

Structural and Multidisciplinary Optimization

Link to article, DOI:

$10.1007 / \mathrm{s} 00158-017-1656-\mathrm{x}$

Publication date:

2017

Document Version

Peer reviewed version

Link back to DTU Orbit

Citation (APA):

Lian, H., Christiansen, A. N., Tortorelli, D. A., Sigmund, O., \& Aage, N. (2017). Combined shape and topology optimization for minimization of maximal von Mises stress. Structural and Multidisciplinary Optimization, 55(5), 1541-1557. https://doi.org/10.1007/s00158-017-1656-x

\section{General rights}

Copyright and moral rights for the publications made accessible in the public portal are retained by the authors and/or other copyright owners and it is a condition of accessing publications that users recognise and abide by the legal requirements associated with these rights.

- Users may download and print one copy of any publication from the public portal for the purpose of private study or research.

- You may not further distribute the material or use it for any profit-making activity or commercial gain

- You may freely distribute the URL identifying the publication in the public portal 


\title{
Combined Shape and Topology Optimization for Minimization of Maximal von Mises Stress
}

\author{
Haojie Lian • Asger N. Christiansen • Daniel A. Tortorelli • Ole \\ Sigmund • Niels Aage
}

November 28, 2017

\begin{abstract}
This work shows that a combined shape and topology optimization method can produce optimal 2D designs with minimal stress subject to a volume constraint. The method represents the surface explicitly and discretizes the domain into a simplicial complex which adapts both structural shape and topology. By performing repeated topology and shape optimizations and adaptive mesh updates, we can minimize the maximum von Mises stress using the $p$-norm stress measure with $p$-values as high as 30 , provided that the stress is calculated with sufficient accuracy.
\end{abstract}

Keywords Stress minimization - Combined shape and topology optimization - Explicit surface representation · Deformable simplicial complex method

Haojie Lian · Niels Aage · Ole Sigmund

Department of Mechanical Engineering, Technical University of Denmark, Nils Koppels Allé, B.404, 2800 Kgs. Lyngby, Denmark

E-mail: naage@mek.dtu.dk

Asger N. Christiansen

Department of Applied Mathematics and Computer Science, Technical University of Denmark, Asmussens Allé, B.303B, 2800 Kgs. Lyngby, Denmark

Daniel A. Tortorelli

Lawrence Livermore National Laboratory, Livermore, CA, USA

\section{Introduction}

The Deformable Simplicial Complex (DSC) method (Misztal and Bærentzen 2012) has recently been applied to structural topology and shape optimization where the objective was to minimize compliance subject to a volume constraint in homogeneous isotropic linear elastic 2D and 3D domains (Christiansen et al 2014a b). However, in many applications, the interest is not to minimize compliance but rather to avoid large stress concentrations which lead to structural fatigue and fracture (Sanford 2003). Consequently, we will apply DSCbased topology and shape optimization to the problem of minimizing the maximal von Mises stress subject to a volume constraint 1

One should note that minimizing the maximal von Mises stress is more difficult than compliance due to the "singularity" phenomenon, its local nature and its highly non-linear behavior (Le et al 2010). Nevertheless, minimizing the maximal von Mises stress has been treated in shape optimization, i.e. with explicit boundary representation in e.g. Francavilla et al (1975); Changwen and Minghua (1990); Le et al (2011). Furthermore, a maximum von Mises stress constraint has been enforced in topology optimization problems (e.g. Duysinx and Sigmund (1998); Svanberg and Werme (2007); Brugpi and Venini (2008); Le et al (2010); Cheng and Jiang (1992); Allaire and Jouve (2008); Allaire et al (2011, 2014); Ha and Cho (2008); Yamasaki et al (2011)) and a number of other papers. In the work of (Amstutz and Novotny 2010) the authors present a topology optimization method which utilizes a $p$-norm stress measure with very high $p$ 's such that the locality of stresses

1 A preliminary version of this work was presented at the 4th International Conference on Engineering Optimization (EngOpt 2014). 
is resolved. Common for the aforementioned topology optimization methods is that they all use implicit representation of boundaries and a smoothed von Mises stress measure for the objectives and/or constraints. In this work, we consider the fundamental question; given a specified amount of material what is the structural layout that leads to the smallest (global) stress? To answer this question we minimize the approximate maximal von Mises stress of a structure with respect to both shape and topology via an explicit boundary representation.

In our DSC approach, the design domain is discretized into non-overlapping triangular elements (a simplicial complex) that are either labeled solid (filled with material) or void (filled with air). Since the initial step is to discretize the design problem, it should be noted that the presented work applies the discretize-then-optimize approach to solving the optimization problem. Consequently, the structural surface is represented explicitly by the collection of edges which are sandwiched between the void and solid elements. Even though the structure is represented explicitly (as opposed to e.g. the density method (Bendsøe 1989, Bendsøe and Sigmund 2003) and level set method (Wang et al 2003; Allaire et al 2004)), the DSC method is able to accommodate large shape and topology changes while maintaining well-shaped triangular elements. It does so by repeatedly performing local mesh operations. Furthermore, this approach uses a single representation of the domain discretization as opposed to combined implicit/explicit approaches as e.g. Allaire et al (2013).

The DSC optimization method (Christiansen et al 2014a b) changes the shape and topology of a structure by staggering discrete and continuous ${ }^{2}$ optimization steps. The discrete step relabels elements from solid to void based on an analytical optimization procedure which uses the topological derivative, or a heuristic measure, whereas the continuous step moves the nodes using classical shape optimization techniques.

To generate a differentiable cost function, the $p$ norm stress is used as an approximation to the maximum function (Duysinx and Sigmund 1998). We show that the $p$-norm stress based on the stress values evaluated in element centroids, i.e. via one point quadrature, results in non-physical jagged surfaces (Haftka and Gürdal 1992). This well-known problem is alleviated by performing a more accurate $p$-norm calculation based on a higher order quadrature.

The method is described in detail in Section 2 and verified on the well-known plate with a hole example. In

\footnotetext{
2 Here, and throughout the paper, the use of "continuous optimization" refers to solving a discretized optimization problem with continuous design variables.
}

section 3 we investigate the influence of the $p$-norm integration order and in section 4, we apply the suggested approach to two 2D problems: the L-shaped cantilever beam (also known as the Norwegian sock) and the portal frame. The results show that the maximal stress is significantly reduced compared to the corresponding compliance optimized designs. We summarize our findings in Section 5 .

\section{Method}

\subsection{Discretization}

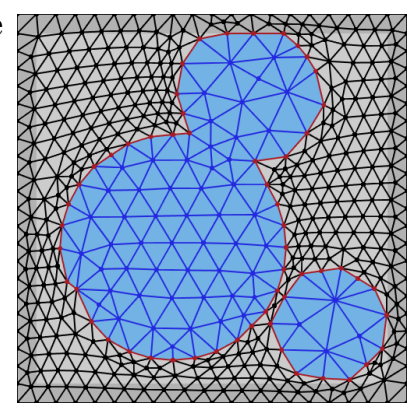

(a) Time step 1

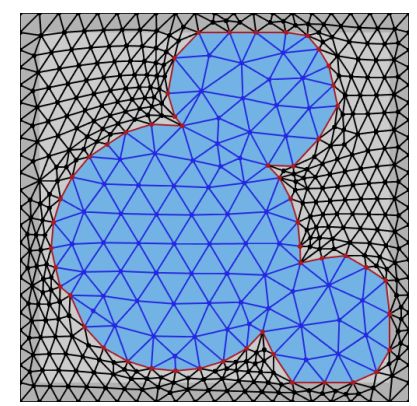

(b) Time step 2
Fig. 1 Example that demonstrates how the DSC method labels solid, void and interface regions and how it performs a topology change. In the figure the white region corresponds to void and blue to solid.

The proposed DSC optimization approach uses a simplicial complex to represent the structure, i.e. it discretizes the design domain into triangular elements as seen in Fig. 1. The elements $e$ consist of either void (no material) or solid (filled with material). Therefore, the interface between solid and void (the surface) is represented by the collection of element edges that are sandwiched between a triangle labeled void and a triangle labeled solid. In addition to serving as the geometric representation, the solid elements of this discretization are also used for the finite element analysis. This means that "void" regions are not included in the FE analysis. To ensure an accurate analysis, it is important to sustain a high quality mesh with no degenerate triangles within the solid region; the mesh quality of the void region is of no concern cf. Fig. 1. Based on an isotropic plane stress assumption, the (linear) finite element equations for the 2D solid domain investigated in this paper is stated as follows

$K u=f$ 
where $\boldsymbol{K}$ is the global stiffness matrix, $\boldsymbol{f}$ is the global load vector and $\boldsymbol{u}$ is the global displacement vector. The element $e$ stiffness matrix and load vector are evaluated as

$$
\begin{aligned}
\boldsymbol{K}^{e} & =\int_{\Omega^{e}} h\left(\boldsymbol{B}^{e}\right)^{T} \boldsymbol{E}^{e} \boldsymbol{B}^{e} \mathrm{~d} \Omega \\
& \approx \sum_{g}^{n_{g}} h\left(\boldsymbol{B}_{g}^{e}\right)^{T} \boldsymbol{E}_{g}^{e} \boldsymbol{B}_{g}^{e} J_{g} w_{g} \\
\boldsymbol{f}^{e} & =\int_{\Gamma^{e}} h\left(\boldsymbol{N}^{e}\right)^{T} \boldsymbol{q}^{e} \mathrm{~d} \Gamma \approx \sum_{g}^{n_{g}} h\left(\boldsymbol{N}_{q}^{e}\right)^{T} \boldsymbol{q}_{g} J_{g} w_{g}
\end{aligned}
$$

where $\boldsymbol{B}^{e}$ is the strain displacement matrix, $\boldsymbol{E}^{e}$ the constitutive matrix, $\boldsymbol{N}^{e}$ the shape function matrix, $h$ is the thickness and $\boldsymbol{q}^{e}$ is the element traction force. Due to its importance in the proposed optimization method, we also state the Gauss integration summation where $g$ refers to the Gauss point index, $n_{g}$ the number of Gauss points, $J_{g}$ the element Jacobian, and $w_{g}$ the weight factor. Throughout the paper we employ quadratic and linear Lagrange shape functions to interpolate the displacements and geometry, respectively. That is, we use straight-sided triangles for the geometry and quadratic representation (6-noded Lagrange triangles) of the physics. This formulation proves advantageous for the shape optimization presented in Sec. 2.2 .2

\subsection{Optimization}

The optimization algorithm follows the approach from (Christiansen et al 2014a b) in the sense that each iteration consists of three distinct steps cf. Fig. 2 flowchart. The first step is to perform a topological design update, i.e. to insert one or more holes as described in the upcoming section 2.2.1. The second step updates the shape by solving a continuous, parameter free shape optimization problem which is presented in section 2.2.2. The first two steps are performed on a fixed mesh and the third step updates the mesh by the DSC method as described in section 2.2.3. Finally, in section 2.2.4. the termination criteria is explained, and suggestions for making the optimization process more efficient are provided.

\subsubsection{Topology update}

Although DSC can change the topology by merging holes or material domains, it still lacks the ability to generate new holes. One remedy to resolve this shortcoming is to initiate the optimization from structures

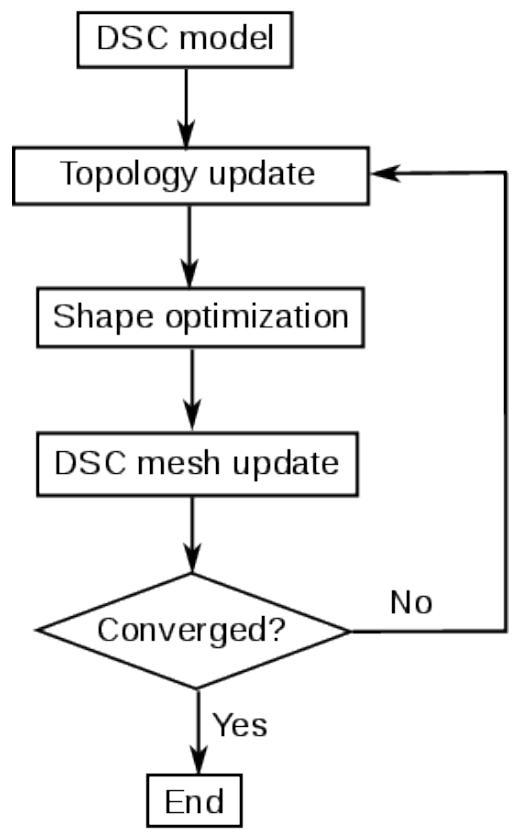

Fig. 2 DSC optimization flowchart.

with many holes, as often used in level-set approaches, but this leads to a dependence on the initial design and restricts the topological search space. Hence, it is more desirable to use a fully solid initial design and introduce holes during the optimization process. In compliance optimization (Christiansen et al (2014b)), holes are introduced using the topological derivative. As an alternative to using the topological derivative for the $p$-norm stress measure, we use the analytical value for a stress concentration around a circular hole in an infinite plate as criterion for hole insertion (Savin 1961). Acknowl-

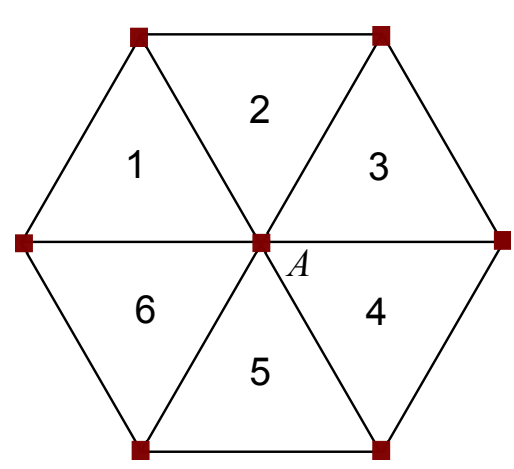

Fig. 3 An element patch centred on node A. The red squares denote element vertices.

edging the fact that the removal of a single element causes stress concentrations, we use a heuristic hole insertion rule that utilizes averaging over small element 
patches as shown in Fig. 3. That is, for all elements we compute the maximum element stress measure $\tilde{\sigma}_{e}$ as

$\tilde{\sigma}_{e}=\max _{g}\left(\left|3 \sigma_{1}^{g}-\sigma_{2}^{g}\right|,\left|\sigma_{1}^{g}+\sigma_{2}^{g}\right|\right)$

where $\sigma_{1}^{g}$ and $\sigma_{2}^{g}$ are the principal stresses at Gauss point $g$ (with $\sigma_{1}^{g} \geq \sigma_{2}^{g}$ ). The nodal averaged stress measure $\tilde{\sigma}_{A}$, associated with node $A$, is the average of the neighbouring elements maximum values (Fig. 3), i.e.

$\tilde{\sigma}_{A}=\frac{\sum_{e} \tilde{\sigma}_{e}}{N_{e}}$

where $N_{e}$ refers to the number of elements which share node $A$, i.e. the number of elements in the node $A$ element patch. The hole insertion step identifies the node with the minimum stress measure $\tilde{\sigma}_{A}$, and removes its corresponding element patch. Note that only one hole can be inserted per FEM analysis. Even though we remove patches rather than single elements we will inevitably introduce stress concentrations. This issue is alleviated by the subsequent shape optimization step.

\subsubsection{Shape optimization}

The shape optimization step updates the structure to a new configuration which is within a small perturbation of its current shape. That is, based on the current mesh configuration, i.e. the discretized system, we use a parameter free shape optimization procedure to determine new positions of the solid and void interface nodes. Note that during this step the mesh topology remains unaltered. The design variables in this optimization step are the normal movements $t_{n}$ of the interface nodes that are not subjected to external loads (Fig. 4). The new positions of the interface nodes are given by

$\boldsymbol{p}_{n}\left(t_{n}\right)=\boldsymbol{p}_{n}^{0}+t_{n} \boldsymbol{n}_{n}$

Here, $\boldsymbol{p}_{n}^{0}$ is the initial position of node $n$ and $t_{n}$ is the design variable. The normal, $\boldsymbol{n}_{n}$, of node $n$ is computed by averaging the normals of the interface elements which are connected to node $n$.

To prevent the supported nodes in moving away from the supported region (line), support nodes can only move tangentially to the support as illustrated in Fig. 4. Note that we still denote this direction by $\boldsymbol{n}_{n}$. Furthermore, only the edge supported nodes are moved during the shape optimization; the interior supported nodes are fixed.

The design variables $t_{n}$ are collected in a vector $\boldsymbol{t}=\left[\ldots, t_{n}, \ldots\right]^{T}$ and the shape optimization problem

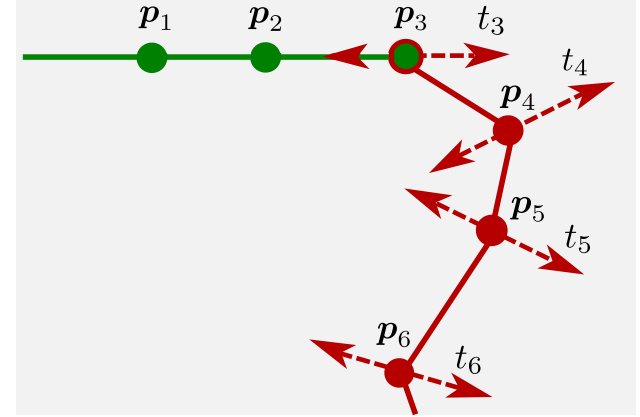

Fig. 4 Supported nodes (green) are not perturbed during the continuous optimization step except the nodes at the boundary of the support (green and red). However, these nodes are moved along the support rather than normal to it, as opposed to the unsupported nodes (red).

is formulated as

$$
\begin{aligned}
\boldsymbol{t}^{*}=\underset{\boldsymbol{t}}{\arg \min }: & \phi(\boldsymbol{p}(\boldsymbol{t}))=\max \sigma(\boldsymbol{p}(\boldsymbol{t})) \\
\text { subject to } & : g(\boldsymbol{p}(\boldsymbol{t}))=\frac{V(\boldsymbol{p}(\boldsymbol{t}))}{V^{*}}-1 \leq 0 \\
: & \boldsymbol{K}(\boldsymbol{p}(\boldsymbol{t})) \boldsymbol{u}(\boldsymbol{p}(\boldsymbol{t}))=\boldsymbol{f} \\
& : \boldsymbol{t}^{\min } \leq \boldsymbol{t} \leq \boldsymbol{t}^{\max }
\end{aligned}
$$

Here, $V(\boldsymbol{p}(\boldsymbol{t}))$ is the volume of the structure and $V^{*}$ is the maximum volume allowed. The equilibrium (FE) equations are restated with design dependence where it is noted that the load is design independent. $\boldsymbol{t}^{\mathrm{min}}$ and $\boldsymbol{t}^{\max }$ are lower and upper limits on the design variables $\boldsymbol{t}$ which are prescribed such that triangles do not degenerate and nodes do not move outside of the design domain during the shape optimization step. Consequently, the magnitude of shape changes is determined by the element size. Apart from their superior FE convergence properties, the use of second order elements for the interpolation of the displacement field also enables the use of larger elements and hence larger shape changes. Note that we use straight-sided triangles as done in Christiansen et al (2014b).

The von Mises stress is evaluated as

$\sigma(\boldsymbol{p}(\boldsymbol{t}))=\sqrt{\boldsymbol{\sigma}^{T}(\boldsymbol{p}(\boldsymbol{t})) \boldsymbol{A} \boldsymbol{\sigma}(\boldsymbol{p}(\boldsymbol{t}))}$,

where $\boldsymbol{A}$ is given by

$\boldsymbol{A}=\left[\begin{array}{ccc}1 & -\frac{1}{2} & 0 \\ -\frac{1}{2} & 1 & 0 \\ 0 & 0 & 3\end{array}\right]$

and $\boldsymbol{\sigma}$ is the stress vector given by

$\boldsymbol{\sigma}(\boldsymbol{p}(\boldsymbol{t}))=\boldsymbol{E B}(\boldsymbol{p}(\boldsymbol{t})) \boldsymbol{u}(\boldsymbol{p}(\boldsymbol{t}))$. 
Since the max function is not smooth, the maximum von Mises stress is replaced by a differentiable approximate expression, e.g. the $p$-norm stress,

$\max \sigma(\boldsymbol{p}(\boldsymbol{t})) \approx\left(\int_{\Omega} \sigma^{p}(\boldsymbol{p}(\boldsymbol{t})) \mathrm{d} \Omega\right)^{\frac{1}{p}}=\phi(\boldsymbol{p}(\boldsymbol{t}))$

where $p$ is relatively "large", cf. Duysinx and Sigmund (1998). The $p$-norm stress, which is always larger than or equal to the true maximum stress, is integrated numerically via

$$
\left(\int_{\Omega} \sigma^{p}(\boldsymbol{p}(\boldsymbol{t})) \mathrm{d} \Omega\right)^{\frac{1}{p}}=\left(\sum_{e}^{n_{e}}\left(\sum_{g}^{n_{g}} \sigma_{g}^{p}(\boldsymbol{p}(\boldsymbol{t})) J_{g}(\boldsymbol{t}) w_{g}\right)\right)^{\frac{1}{p}}
$$

where $n_{e}$ denotes the number of solid elements. A close look at the expression for the $p$-norm stress integration reveals an obvious and extremely important requirement to the numerical scheme. Since $p$ needs to take large values, the $\sigma^{p}$ field within an element may be highly non-linear and cannot be recovered accurately by a single point quadrature $\left(n_{g}=1\right)$. In theory, $\sigma$ is of order 1 for quadratic elements which means that $\sigma^{p}$ with $p=20$ would require the use of $n_{g}=79$ Gauss points for exact integration. This number of Gauss point could have been used in the presented work, but as our numerical experiments of Section 4 will show, we have found that $n_{g}=3$ works in most cases and that seven Gauss points $\left(n_{g}=7\right.$, see Fig. 5) is enough to ensure robustness for the considered design problems.

With the proposed higher order quadrature, we eliminate the need for shape filtering (cf. Le et al (2011); Bletzinger (2014)). A filter may still be desirable if additional boundary smoothness is desired (however, it is noted that its implementation is cumbersome at best). In general, a filter is also used to reduce complexity of designs in topology optimization. Here, we limit the design complexity indirectly by placing the bound on the minimum element size parameter $\delta_{\min }$.

The shape optimization problem is solved by the Method of Moving Asymptotes (MMA), cf. (Svanberg 1987). Convergence is deemed if the Karush-Kuhn-Tucker conditions are satisfied to within an acceptable tolerance, if the mesh becomes excessively distorted or if the maximum number of iterations $N_{\max }=20$ is exceeded, whence the overall optimization process described in the flow chart from Fig. 2 continues.

Due to the stress concentrations which appear in regions surrounding concentrated loads and supports, we omit them when evaluating the objective function. This is justifiable since such loadings and corners are idealizations of distributed loads and small fillets and

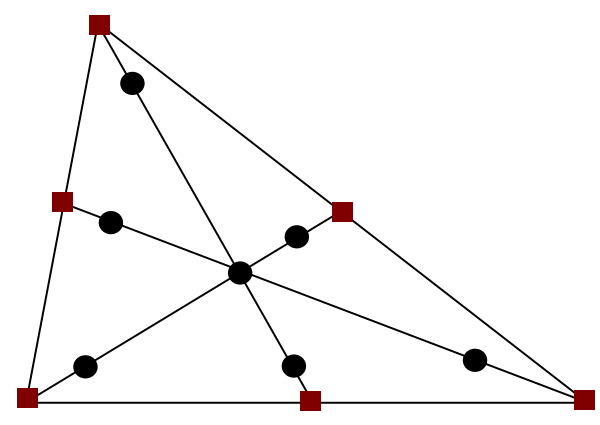

Fig. 5 Distribution of the seven Gauss points used for the $p$ norm stress integration. The red squares denote the element nodes, and the black dots denote the Gauss points.

rounds. In our implementation, we simply exclude element contributions to the element summation in Eq. (12) if the element center is within a small distance of these features.

\subsubsection{Mesh update by the Deformable Simplicial Complex method}

The last step in the design cycle is to update the mesh resulting from the shape optimization, and if necessary, to merge material (or void) domains if they are sufficiently close to one another cf. Fig 1. For this purpose the DSC method is used (Misztal and Bærentzen $2012)^{3}$. The use of DSC for combined shape and topology optimization, i.e. the necessary mesh operations, is explained in details in (Christiansen et al 2014b) and the following should be regarded as a brief summary of these findings.

The DSC method removes low quality triangles (caps and needles) by performing mesh operations such as Laplacian smoothing, edge flip, vertex insertion and vertex removal. Note that the DSC method only improves the mesh quality where necessary (often near the surface) and, as a consequence, it is much faster than a complete remeshing. The DSC method also performs mesh topology changes by collapsing low quality triangles which are sandwiched between two surfaces.

In addition to ensuring high quality elements, the DSC method also controls the level of detail of both the surface and the mesh by collapsing small triangles and splitting large triangles. Consequently, we always attain a mesh of the desired complexity, described by the discretization parameter $\delta_{\text {ave }}$ (corresponding to the average element edge length in the material mesh). More importantly, the DSC method allows for mesh adaptivity so that regions over which the response is smooth are represented by a coarser discretization and vica versa.

3 An open-source framework is available at ww.github. com/asny/2D-DSC 
This is especially relevant in designs with small scale features which lead to large stress concentrations.

\subsubsection{Problem stages and stopping criteria}

Our hybrid optimization scheme requires at least two global convergence criteria to ensure that the process is not stopped prematurely. That is, at least one hole insertion and shape optimization step. In addition to these criteria, we also monitor whether or not the volume constraint is fulfilled and the final topology i.e number of holes, is reached. Since we start from a fully solid design domain the goal is to reach the active volume constraint as fast as possible. For this reason it is undesirable to perform too many shape optimizations since these only make small volume perturbations. On the other hand, hole insertions quickly reduce volume. Using this as a rule of thumb and requiring that the process be stable, we proceed as follows:

While the final volume fraction constraint is not reached, the active volume fraction constraint is relaxed by subtracting $2 \%$ from the previous active volume fraction. This is similar to (B)ESO type approaches that also often start from a fully solid design (Querin et al 1998). Repeated hole insertion steps (i.e. one finite element analysis for each patch that is removed) are performed, until the new active volume fraction constraint is reached. This is followed by a single shape optimization step and a DSC update (as shown in Fig. 2). This multiple hole insertion process continues until the final volume fraction constraint is reached. The $2 \%$ is chosen to render the method robust; increasing this number will hasten the optimization process considerably, however, at the risk of losing stability.

Once the final volume fraction constraint is active we must decide when to stop the hole insertion process. Hole insertions cease when a hole is introduced in the same location in two succesive iterations. More specifically we insert a hole and perform 15 consecutive shape optimization steps and DSC updates. Based on our numerical experiments the 15 steps are sufficient to allow the hole to merge or expand as necessary. After the 15 shape optimization steps are completed the next hole is inserted and its location is compared to that of the previously inserted hole. The process terminates when the new hole is located over a previously merged hole. The tolerance for determining the hole proximity is $\left\|\boldsymbol{c}_{\text {old }}-\boldsymbol{c}_{\text {new }}\right\|_{2} \leq 2 \delta_{\text {ave }}$, where $\boldsymbol{c}_{\text {new }}$ and $\boldsymbol{c}_{\text {old }}$ are the patch center positions for the current and previous hole. From this condition it is clear that the hole proximity detection procedure is mesh dependent.

After the hole insertion ends we assume that the final topology is reached. The remainder of the opti- mziation process only performs shape optimization and DSC steps until convergence at which time the whole optimization algorithm is stopped. This convergence occurs when the change in nodal positions between consecutive shape optimization steps fullfills $\| \boldsymbol{p}_{\text {old }}^{S H A P E}-$ $\boldsymbol{p}_{\text {new }}^{\text {SHAPE }} \|_{\infty}<0.0125 \delta_{\text {ave }}$ and the change in nodal positions between consecutive DSC updates fulfills $\| \boldsymbol{p}_{\text {old }}^{D S C}$ $\boldsymbol{p}_{\text {new }}^{D S C} \|_{\infty}<0.67 \delta_{\text {ave }}$. We remark the 0.0125 and 0.067 values are based on numerical experiments and that they are chosen conservatively such that they ensure the optimization process does not stop prematurely. Note that this need not be the case as we can replace $\delta_{\text {ave }}$ with a mesh independent parameter. This is however deemed outside the scope of this manuscript.

\subsection{Sensitivity analysis}

The non-linear optimization problem (Eq. 7 with the objective function replaced by Eq. (12) ) is solved for $\boldsymbol{t}^{*}=\left[\ldots, t_{n}^{*}, \ldots\right]^{T}$ using the gradient-based optimization algorithm Method of Moving Asymptotes (MMA) (Svanberg 1987). We compute the necessary derivatives of the objective and constraint functions with respect to each design variable $\boldsymbol{t}$ using the adjoint method. For the $p$-norm, the element adjoint load is obtained

$$
\boldsymbol{F}_{\lambda}^{e}=\phi(\boldsymbol{p}(\boldsymbol{t}))^{1-p} \int_{\Omega^{e}} \sigma(\boldsymbol{p}(\boldsymbol{t}))^{p-1}\left(\frac{\partial \sigma(\boldsymbol{p}(\boldsymbol{t}))}{\partial \boldsymbol{u}} \mathrm{d} \Omega\right)^{T}
$$

with

$$
\begin{aligned}
\frac{\partial \sigma(\boldsymbol{p}(\boldsymbol{t}))}{\partial \boldsymbol{u}} & =\frac{\partial \sqrt{\boldsymbol{\sigma}(\boldsymbol{p}(\boldsymbol{t}))^{T} \boldsymbol{A} \boldsymbol{\sigma}(\boldsymbol{p}(\boldsymbol{t}))}}{\partial \boldsymbol{u}} \\
& =\frac{\boldsymbol{\sigma}(\boldsymbol{p}(\boldsymbol{t}))^{T} \boldsymbol{A} \boldsymbol{E} \boldsymbol{B}(\boldsymbol{p}(\boldsymbol{t}))}{\sigma(\boldsymbol{p}(\boldsymbol{t}))}
\end{aligned}
$$

After solving the adjoint problem for $\boldsymbol{\lambda}$, the gradient is computed from

$$
\begin{aligned}
\frac{\partial \phi(\boldsymbol{p}(\boldsymbol{t}))}{\partial t_{n}} & =-\boldsymbol{\lambda}^{T} \frac{\partial \boldsymbol{K}(\boldsymbol{p}(\boldsymbol{t}))}{\partial t_{n}} \boldsymbol{u}(\boldsymbol{p}(\boldsymbol{t}))+\frac{1}{p} \phi^{1-p}(\boldsymbol{p}(\boldsymbol{t})) \times \\
& \sum_{e}^{N_{e}} \int_{\tilde{\Omega}_{e}}\left(p \sigma(\boldsymbol{p}(\boldsymbol{t}))^{p-1} \frac{\partial \sigma(\boldsymbol{p}(\boldsymbol{t}))}{\partial t_{n}} J(\boldsymbol{p}(\boldsymbol{t}))\right. \\
& \left.+\sigma(\boldsymbol{p}(\boldsymbol{t}))^{p} \frac{\partial J(\boldsymbol{p}(\boldsymbol{t}))}{\partial t_{n}}\right) \mathrm{d} \tilde{\Omega}_{e}
\end{aligned}
$$

where $\tilde{\Omega}_{e}$ is the parent element. That is, all elements sharing node $n$ contributes to its sensitivity, c.f. Fig. 3 
The derivative of the stress measure is given as

$$
\begin{aligned}
\frac{\partial \sigma(\boldsymbol{p}(\boldsymbol{t}))}{\partial t_{n}} & =\frac{\partial \sqrt{\boldsymbol{\sigma}(\boldsymbol{p}(\boldsymbol{t}))^{T} \boldsymbol{A} \boldsymbol{\sigma}(\boldsymbol{p}(\boldsymbol{t}))}}{\partial t_{n}} \\
& =\frac{\boldsymbol{\sigma}(\boldsymbol{p}(\boldsymbol{t}))^{T} \boldsymbol{A} \boldsymbol{E} \frac{\partial \boldsymbol{B}(\boldsymbol{p}(\boldsymbol{t}))}{\partial t_{n}} \boldsymbol{u}(\boldsymbol{p}(\boldsymbol{t})}{\sigma(\boldsymbol{p}(\boldsymbol{t}))}
\end{aligned}
$$

After the optimized design variables $\boldsymbol{t}^{*}$ are computed, the mesh is deformed via Eq. (6) and updated via the DSC method.

\section{Accuracy and integration order}

The exponent in the $p$-norm stress and the order of the numerical integration rule is critical for the shape optimization. The governing equations are solved, as mentioned earlier, by the finite element method using quadratic elements. This element choice has previously prevented the appearance of jagged surfaces during the continuous optimization step when minimizing compliance (Christiansen et al 2014b). To illustrate how the $p$-norm von Mises stress minimization problem differs from the minimum compliance problem, we study the biaxially loaded plate design problem shown in Fig. 6 .

The design is initialized as shown in Fig. 6 and a maximum volume constraint $V^{*}$ of $80 \%$ of the design domain is enforced. When minimizing compliance, the result is a smooth round hole (Fig. 7), which we know is optimal from analytical theory (Savin 1961). Note, however, that the hole is not completely circular and that the maximum stress is higher than predicted by the theory that assumes an infinite plate. Hence, the comparison was made to show the qualitative aspect of our results. In this plot and in those of Figs. 8, 10 the element stress is defined as the maximum stress attained at the element Gauss points.

Now we minimize the $p$-norm stress integrated with a simple one point quadrature $\left(n_{g}=1\right)$. As seen in Fig. 8 the surface is less smooth already for $p=1$, and for increasing $p$ values it becomes increasingly jagged, creating reentrant corners with stress singularities which are obviously not optimal. To alleviate the jaggedness, the literature has applied filtering techniques to smooth the surfaces. However, here we show that the need for filtering can be eliminated by evaluating the stress norm using higher order quadrature. Already for $n_{g}=3$ we obtain much smoother surfaces as seen in Fig. 9. However, in some cases we still see instabilities e.g. for $p=$

\begin{tabular}{|c||c||c|c|c|}
\hline Objective & $p$ & $\boldsymbol{u}^{T} \boldsymbol{K} \boldsymbol{u}$ & $\max _{i} \sigma_{i}$ & $\left(\int_{\Omega} \sigma^{12} \mathrm{~d} \Omega\right)^{\frac{1}{12}}$ \\
\hline \hline Compliance & - & 609466 & 2.631 & 5.431 \\
\hline$p$-norm stress & 1 & 609739 & 2.783 & 5.476 \\
\hline$p$-norm stress & 4 & 609596 & 2.763 & 5.437 \\
\hline$p$-norm stress & 8 & 609505 & 2.737 & 5.431 \\
\hline$p$-norm stress & 12 & 609478 & 2.627 & 5.429 \\
\hline
\end{tabular}

Table 1 Summary of results from Figs. 7 and 10 Columns give compliance $\left(\boldsymbol{u}^{T} \boldsymbol{K} \boldsymbol{u}\right)$, maximal von Mises stress evaluated at element Gauss points $\left(\max _{i} \sigma_{i}\right)$, and $p$-norm von Mises stress $\left(\left(\int_{\Omega} \sigma^{12} \mathrm{~d} \Omega\right)^{\frac{1}{12}}\right)$ for the test cases optimized for compliance and varying $p$-values using $n_{g}=7$.

12 in Fig. 9p. Next we use $n_{g}=7$ for $p=1,4,8,12$. As shown in Fig. 10 the optimized shapes are smooth for all $p$ values tested. Further, as shown in Tab. 1, the maximum von Mises stress decreases with increasing $p$, which suggests that a proper $p$ value combined with accurate integration, is an effective way of decreasing the maximum von Mises stress. Note also that the compliance minimization also exhibits low stress which is not always the case as will be seen in the subsequent examples.

As a final validation, we modify the problem in Fig. 6 by applying $-\sigma_{2}$ rather than $\sigma_{2}$ which corresponds to a pure shear loading. A maximum volume constraint $V^{*}$ of $90 \%$ of the design domain is enforced. According to the analytical solution (Sternberg and Koiter 1958; Cherkaev et al 1998), the optimized hole should have a sharp corner with the angle equal to $102.6^{\circ}$. Our numerical optimization of the $p$-norm stress $\left(p=12, n_{g}=7\right)$ yields $101.7^{\circ}$ (Fig. 11). Obviously, sharp corners do not appear if a shape smoothing filter is applied 4 We remark that the presence of sharp corners in stress optimized structures is suspicious. Nevertheless, the theory shows that the stress is bounded at a sharp corner. Considering multiple loading cases obviously removes such sharp features.

\section{Results}

In the following examples we use seven point quadrature $\left(n_{g}=7\right)$ for the $p$-norm computation. In each figure we plot the maximum Gauss point von Mises stress of the element and use a color map scaled between the minimum and maximum stress for all subfigures in a given plot. The maximum and minimum edge lengths control the element size along the domain boundary and the maximum and minimum areas control the element size in the interior. The degeneration angle, the degeneration area and the degeneration edge

4 The design with a sharp corner and filtering of other parts in Le et al (2011) obtained by applying a nonuniform filter. 


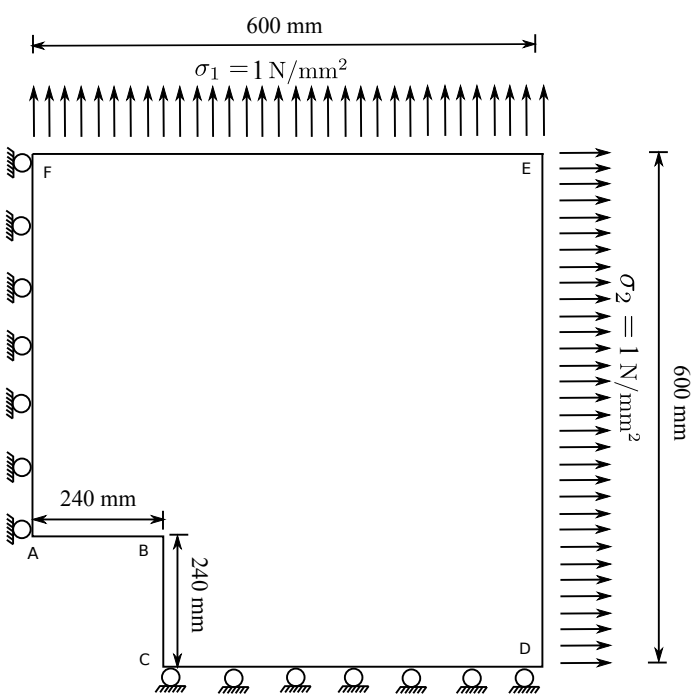

(a) Plate with a hole problem definition

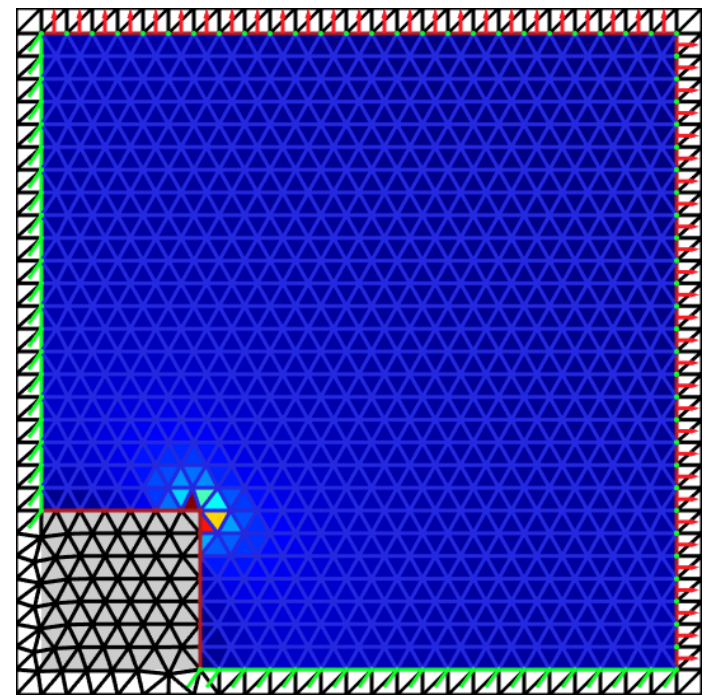

(b) Plate with a hole discretization

Fig. 6 Plate problem definition (left) and the initial discretization (right). The plate thickness is $1 \mathrm{~mm}$. The average edge length is $\delta_{\text {ave }}=20 \mathrm{~mm}$. The "jet" color map is used to visualize the von Mises stress across the structure. The gray color represents the void region. The color scale ranges from $0.84 \mathrm{~N} / \mathrm{mm}^{2}$ to $6.14 \mathrm{~N} / \mathrm{mm}^{2}$.

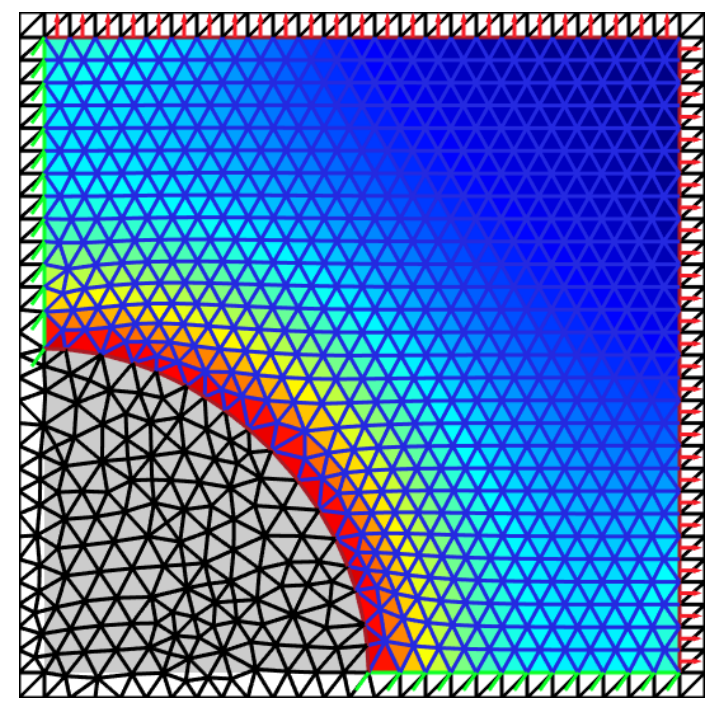

Fig. 7 Hole in a plate design with compliance as the optimization objective. The "jet" color represents the von Mises stress. The color scale ranges from $0.986 \mathrm{~N} / \mathrm{mm}^{2}$ to $2.783 \mathrm{~N} / \mathrm{mm}^{2}$

length (see Christiansen et al (2014a b) for details) are used to maintain the mesh quality. The minimum angle controls the edge flipping in the DSC step and the degeneration angle controls the mesh distortion in the shape optimization step. The DSC settings are shown in Tab. 25 . For all examples we illustrate the design domain by dark gray and include a surrounding border of non-design elements in light grey. This surrounding border is convenient, since it allows objects to extend to

5 For all studies we use $\delta_{\text {ave }}=15 \mathrm{~mm}$ and $A=$ $0.5 \sqrt{3 \delta_{\text {ave }}^{2} / 4} \mathrm{~mm}^{2}$ unless otherwise stated. the boundary of the design domain without any mesh complications cf. (Christiansen et al 2014b). Note that these non-design elements are not included in the FE analysis..

It is noted that maximum stress minimization problems with high $p$-values are extra sensitive to small shape variations and that GCMMA (Svanberg 2002) could be beneficial to avoid oscilations in the optimiza- 


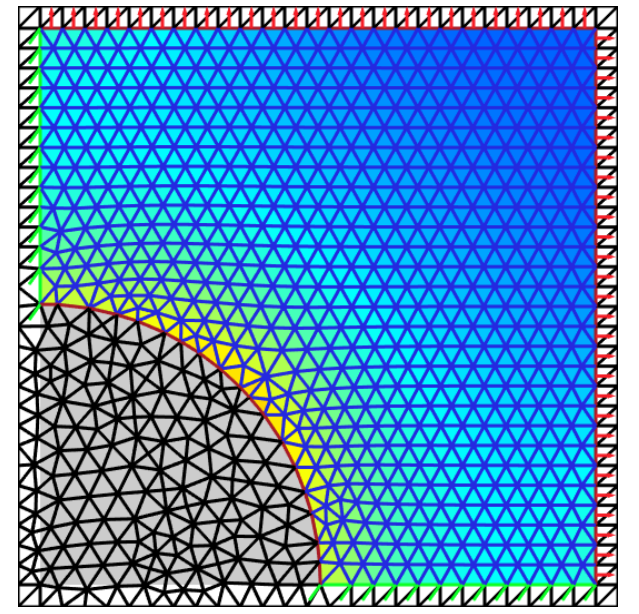

(a) $p=1, n_{g}=1$

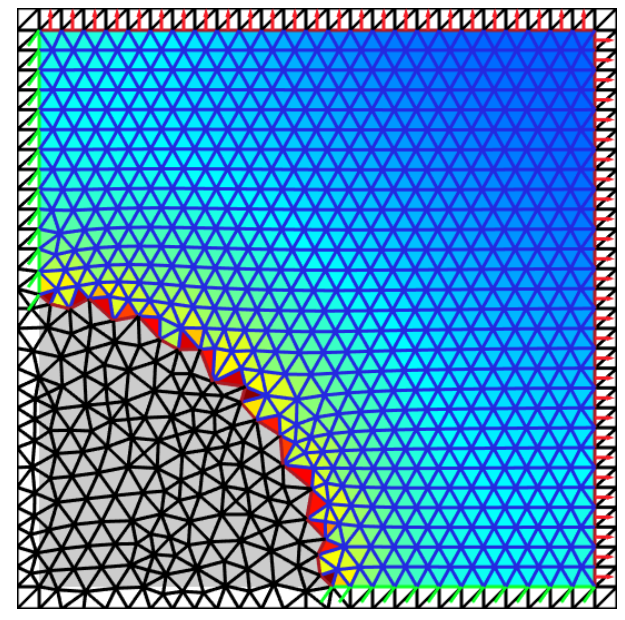

(c) $p=8, n_{g}=1$

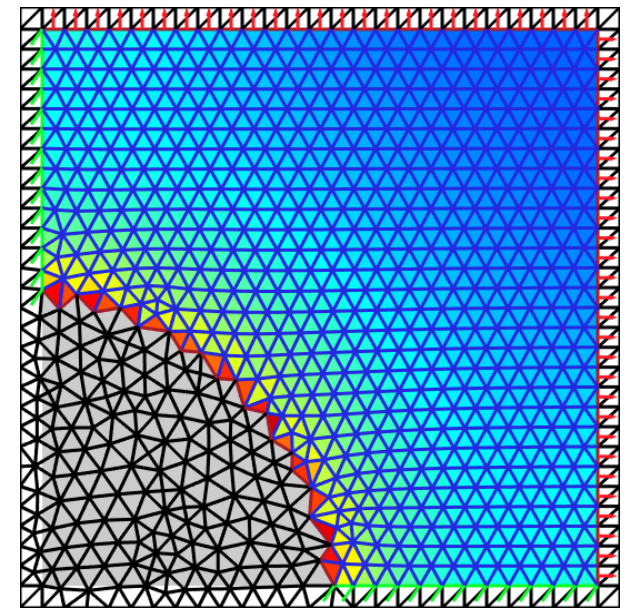

(b) $p=4, n_{g}=1$

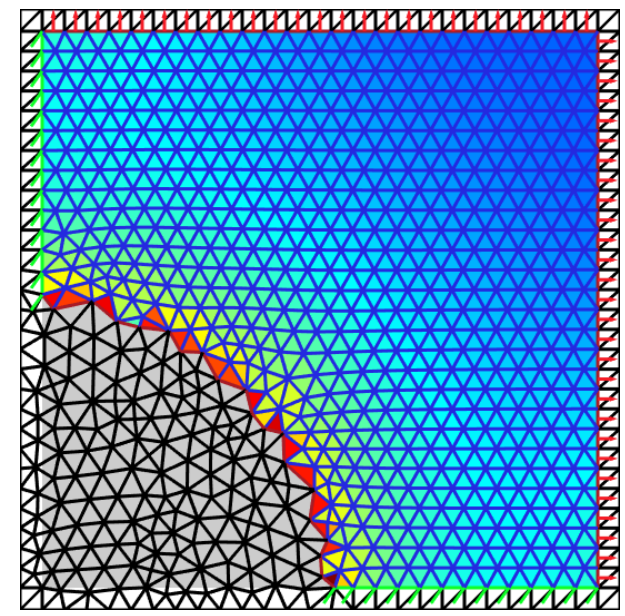

(d) $p=12, n_{g}=1$

Fig. 8 Hole in a plate designs with one point integration for different values of $p$. The "jet" color represents the von Mises stress. The color scale ranges from $0.092 \mathrm{~N} / \mathrm{mm}^{2}$ to $4.167 \mathrm{~N} / \mathrm{mm}^{2}$.

\begin{tabular}{|c|c|c|}
\hline Max area & Min area & Degeneration area \\
\hline $2 A$ & $0.5 A$ & $0.25 A$ \\
\hline \hline Max edge & Min edge & Degeneration edge \\
\hline $2 \delta_{\text {ave }}$ & $0.5 \delta_{\text {ave }}$ & $0.25 \delta_{\text {ave }}$ \\
\hline \hline Min angle & Degeneration angle & Degeneration angle in MMA \\
\hline $20^{\circ}$ & $10^{\circ}$ & $5^{\circ}$ \\
\hline
\end{tabular}

Table 2 DSC mesh settings. A: average element area. $\delta_{\text {ave }}$ : average element edge length.

tion history. That said, we have successfully used MMA with conservative asymptote initializations.

\subsection{L-bracket}

We first study the L-bracket problem (Duysinx and Bendsøe 1998) which is defined and initialized as depicted in Fig. 12. The design problem is to minimize the $p$-norm stress subject to a volume constraint $V^{*}$ of $50 \%$ of the design domain.

We first investigate the effect of the norm parameter $p$ as seen in Figs. 13,14 and Tab. 3. From Fig. 13 we see a more uniform distribution of von Mises stress and a reduced maximal von Mises stress as $p$ is increased. Furthermore, we note that the compliance increases significantly as we increase $p$; unlike the hole in a plate design example. In Fig. 14, we plot the nodal von Mises stress over the edge which connects the support to the load, initially defined as the lines connecting points EDC in Fig. 12. The nodal stress is obtained by element averaging over the nodal von Mises stress for all neighboring elements. Similar to the previous observations, the nodal von Mises stress decreases with increasing $p$. For $p=30$, a very smooth stress distribution is reached. It was surprising being able to ef- 


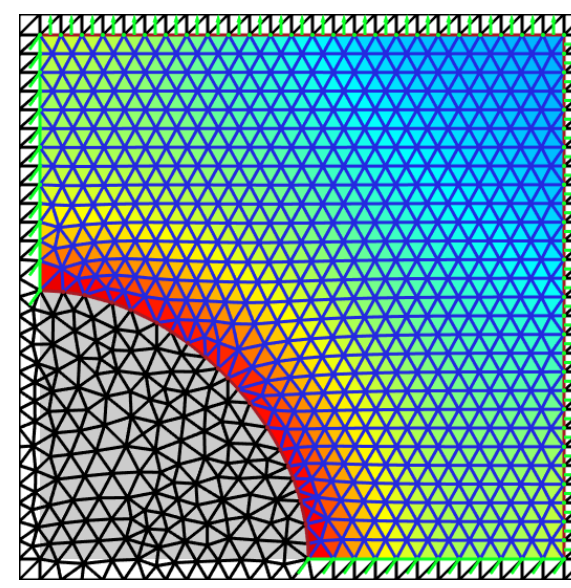

(a) $p=8, n_{g}=3$

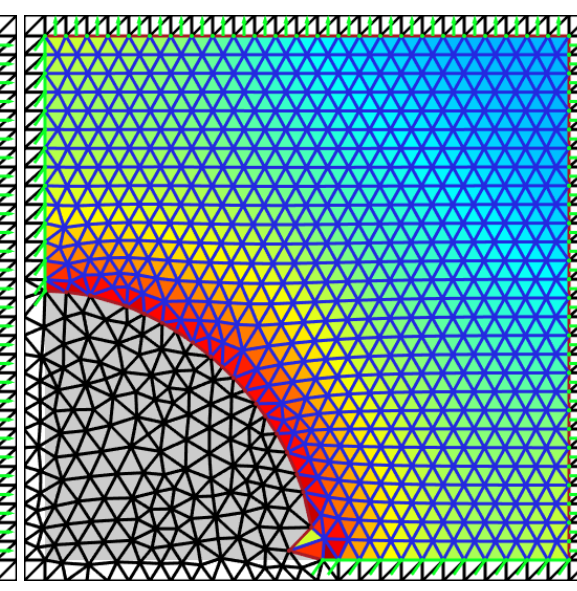

(b) $p=12, n_{g}=3$

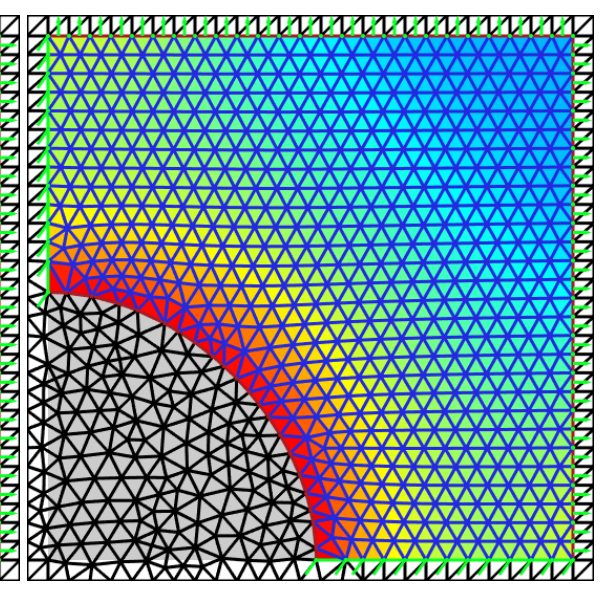

(c) $p=16, n_{g}=3$

Fig. 9 Hole in a plate designs with three point quadrature for different values of $p$. The "jet" color represents the von Mises stress. The color scale ranges from $0.17 \mathrm{~N} / \mathrm{mm}^{2}$ to $2.89 \mathrm{~N} / \mathrm{mm}^{2}$.

fectively optimize with such a high $p$-value, since the $p$-norm is highly ill-conditioned in this case. The success is attributed to the conservative MMA asymptote update strategy which, however, necessitates many design iterations. Despite this drawback we use $p=30$ in the remaining examples.

Not surprisingly, the stress minimization designs result in much more evenly distributed stress compared to the compliance minimized design. The results in Tab. 3 confirm this. Interestingly, the compliance for the stress based designs increase by only $20 \%$, whereas the stress reduces significantly, indicating that one can reduce stress levels without significantly increasing compliance.

Next, we repeat the example with different average element edge lengths ( $\delta_{\text {ave }}=15,12,8 \mathrm{~mm}$ ); all other things being equal. From Fig. 15, it is seen that though different $\delta_{\text {ave }}$ values produce different designs, the overall shape and stress distributions of the problematic corner are similar. The differences in design topologies and shapes clearly show that the proposed methodology is mesh dependent. This is expected since the only length scale control is through the DSC $\delta_{\text {ave }}$ mesh-size parameter. From Tab. 3 it is seen that the design with $\delta_{\text {ave }}=8 \mathrm{~mm}$ has larger maximum von Mises stress than those corresponding to $\delta_{\text {ave }}=12 \mathrm{~mm}$ and $\delta_{\text {ave }}=15 \mathrm{~mm}$. This is expected since mesh refinement leads to a more flexible structure and the Gauss points, where we evaluate the $p$-norm stress, are closer to the boundary, resulting in higher stress values. Fig. 16 which shows that the optimized stress distributions along the inside edge confirms this conjecture. Nonetheless, the stress values for the different designs are similar which means we can obtain satisfactory results without resorting to extremely refined meshes.

\begin{tabular}{|c||c|c||c|c|c|}
\hline Objective & $p$ & $\delta_{\text {ave }}$ & $\boldsymbol{u}^{T} \boldsymbol{K} \boldsymbol{u}$ & $\max _{i} \sigma_{i}$ & $\left(\int_{\Omega} \sigma^{30} \mathrm{~d} \Omega\right)^{\frac{1}{30}}$ \\
\hline \hline Compliance & - & 15 & 598228 & 12.4471 & 13.3571 \\
\hline$p$-norm stress & 4 & 15 & 617983 & 9.18918 & 9.82297 \\
\hline$p$-norm stress & 8 & 15 & 669411 & 5.70153 & 6.20554 \\
\hline$p$-norm stress & 12 & 15 & 706759 & 4.99254 & 5.49015 \\
\hline$p$-norm stress & 30 & 15 & 722620 & 4.23616 & 5.09667 \\
\hline$p$-norm stress & 30 & 12 & 721513 & 4.18581 & 4.99195 \\
\hline$p$-norm stress & 30 & 8 & 763997 & 4.26196 & 5.13208 \\
\hline
\end{tabular}

Table 3 Data for the L-bracket problem with compliance $\left(\boldsymbol{u}^{T} \boldsymbol{K} \boldsymbol{u}\right)$, maximal von Mises stress $\left(\max _{i} \sigma_{i}\right)$ and $p$-norm von Mises stress $\left(\left(\int_{\Omega} \sigma^{30} \mathrm{~d} \Omega\right)^{\frac{1}{30}}\right)$ values optimized for different $p$ and $\delta_{\text {ave }}$.

\begin{tabular}{|c||c|c|c|}
\hline Hole number & $\boldsymbol{u}^{T} \boldsymbol{K} \boldsymbol{u}$ & $\max _{i} \sigma_{i}$ & $\left(\int_{\Omega} \sigma^{30} \mathrm{~d} \Omega\right)^{\frac{1}{30}}$ \\
\hline \hline 0 & 162419 & 8.04827 & 9.73099 \\
\hline 3 & 824016 & 4.62809 & 5.72415 \\
\hline 5 & 741356 & 4.39659 & 5.25673 \\
\hline 16 & 727802 & 4.23505 & 5.10675 \\
\hline
\end{tabular}

Table 4 Results for the L-bracket problem with different initial topology from Figs. 19,22 The table presents compliance $\left(\boldsymbol{u}^{T} \boldsymbol{K} \boldsymbol{u}\right)$, maximal von Mises stress on Gauss points $\left(\max _{i} \sigma_{i}\right)$ and $p$-norm von Mises stress $\left(\left(\int_{\Omega} \sigma^{30} \mathrm{~d} \Omega\right)^{\frac{1}{30}}\right)$ values optimized with $p=30$ and $\delta_{\text {ave }}=15$.

Fig. 17 shows the convergence histories of the $p$ norm stress and volume for a specific optimization run. 


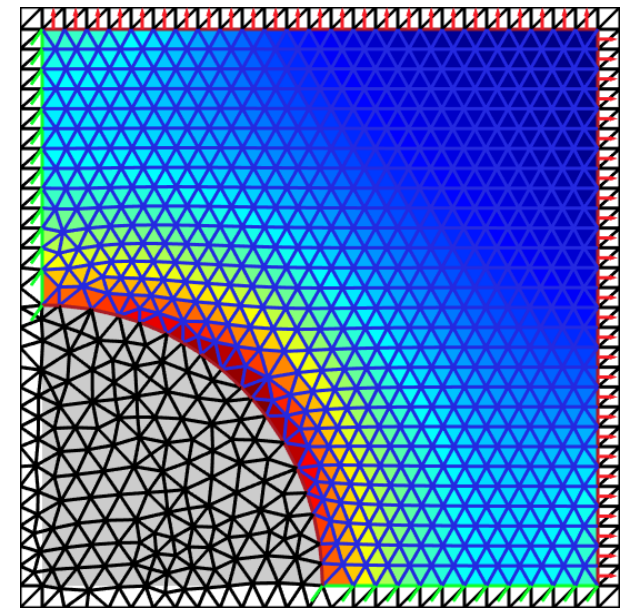

(a) $p=1, n_{g}=7$

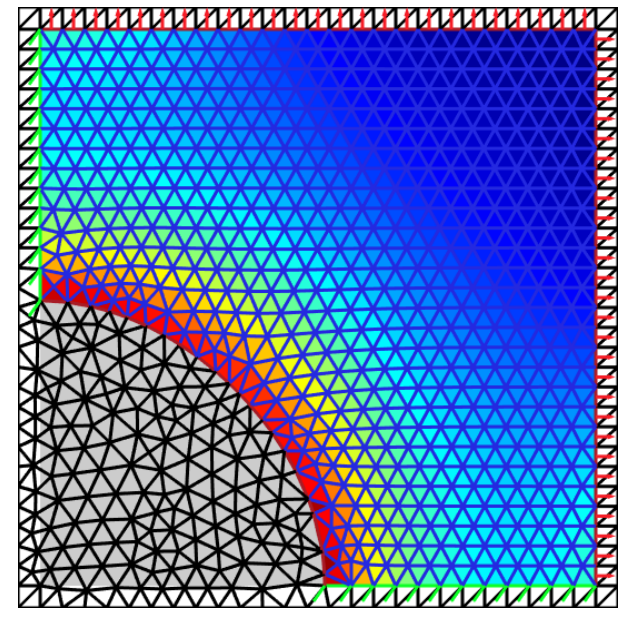

(c) $p=8, n_{g}=7$

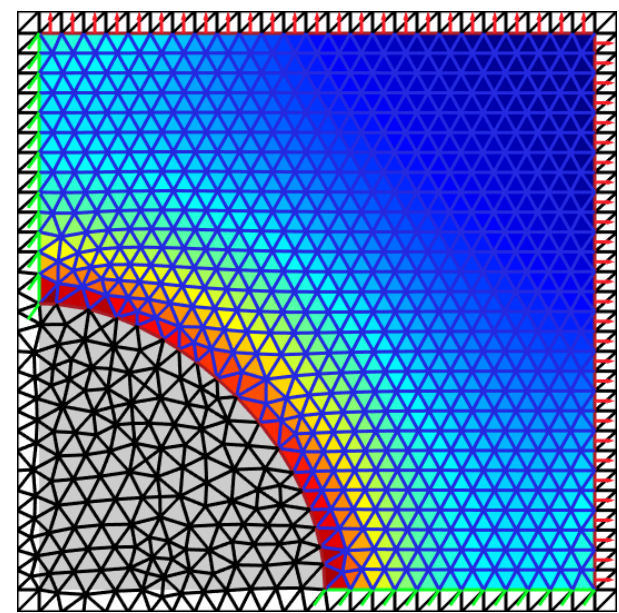

(b) $p=4, n_{g}=7$

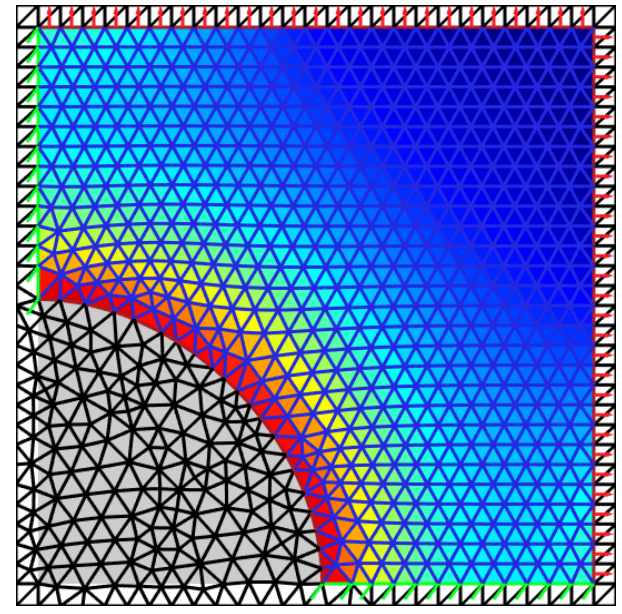

(d) $p=12, n_{g}=7$

Fig. 10 Hole in a plate designs for minimization of stress with seven point quadrature $\left(n_{g}=7\right)$ for different values of $p$. The "jet" color represents the von Mises stress. The color scale ranges from $0.986 \mathrm{~N} / \mathrm{mm}^{2}$ to $2.783 \mathrm{~N} / \mathrm{mm}^{2}$.

The oscillations are attributed to the DSC updates that change mesh and structural topology. However, as the design process converges and holes are no longer inserted, the oscillations cease. We emphasize that the total number of FE evaluations is high compared to other topology optimization approaches, albeit some efficiency is achieved by using fewer high-order elements to represent the design.

To verify the optimality of the optimized designs the $\delta_{\text {ave }}=15 \mathrm{~mm}$ design is studied. For a single constraint problem the KKT conditions state that $\nabla f+\lambda \nabla g=0$ for all non-bounded design variables. This is illustrated in Fig. 18 which shows the KKT condition for the nonbounded variables in the direction of negative $\nabla_{i} g$ with lengths scaled by $\nabla_{i} f / \nabla_{i} g$. The figure shows that the arrows have (almost) equal lengths and thus serves as a visual proof that the optimality condition is close to being satisfied for this design.

Another observation from Fig. 18 is that the stress along the boundaries is far from uniform. Hence, a fullystressed design strategy, which adds material at highly stressed boundaries and subtracts at low stressed boundaries, would lead to an entirely different (and sub optimal) topology. The difference between the fully-stressed design strategy and the strategy used here is that the former does not rely on sensitivity information, and hence results in suboptimal designs; our designs satisfy the optimality conditions.

To further validate the hole insertion step, we compare our design with $p=30$ and $\delta_{\text {ave }}=15$ of Fig. 13 to shape only optimized designs. Figs 19 -22 show the shape only designs corresponding to different initial topologies. Bear in mind, the DSC can still change the 


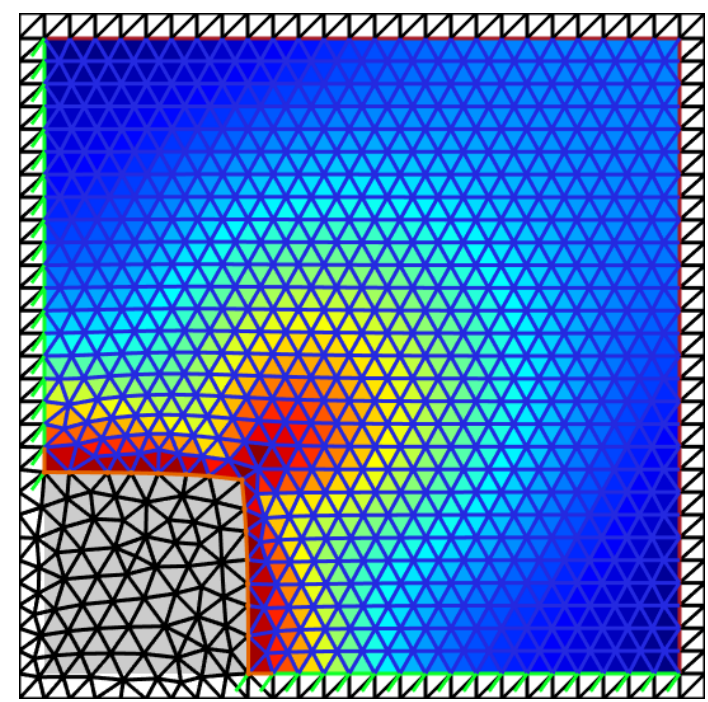

Fig. 11 Hole in a plate designs subject pure shear. The $p$-norm stress $(p=12)$ is the optimization objective. The "jet" color represents the von Mises stress. The color scale ranges from $0.87 \mathrm{~N} / \mathrm{mm}^{2}$ to $4.51 \mathrm{~N} / \mathrm{mm}^{2}$.

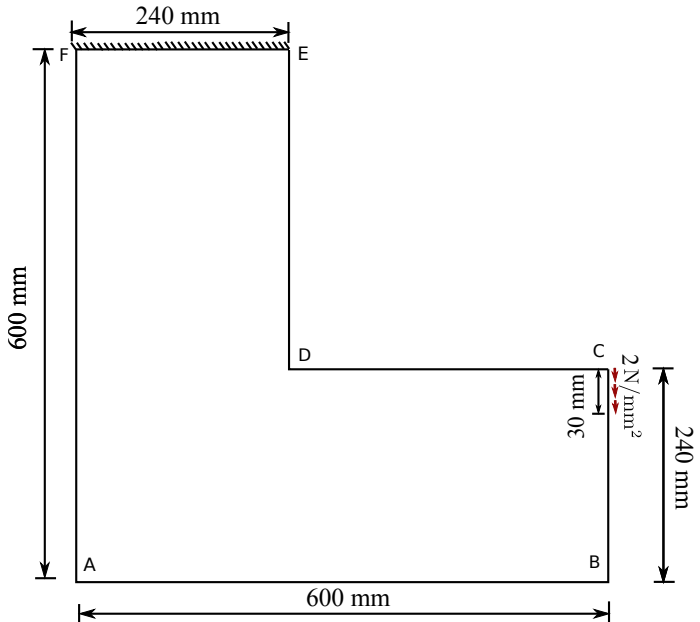

(a)

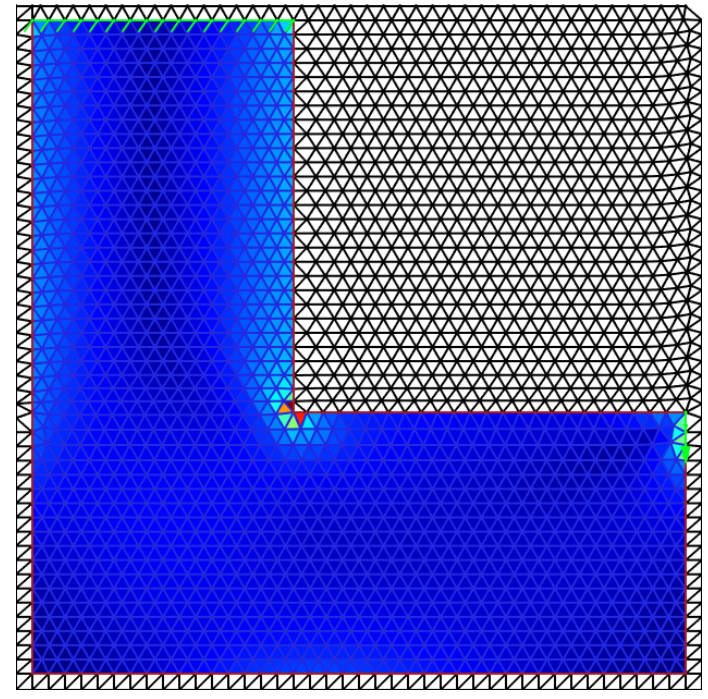

(b)

Fig. 12 L-bracket problem definition (a) and the initial discretization (b). The L-bracket thickness is $1 \mathrm{~mm}$ and the average edge length $\delta_{\text {ave }}=15 \mathrm{~mm}$. The "jet" color map represents the von Mises stress. The color scale ranges from $0.02 \mathrm{~N} / \mathrm{mm}^{2}$ to $14.11 \mathrm{~N} / \mathrm{mm}^{2}$.

topology by merging holes. As expected, the optimized designs depend on the initial topology and the design corresponding to the initial structure without holes is the worst Finally, it is observed that none of the shape only designs perform as well as the topology optimized design.

\subsection{Portal}

The last example considers the design of a portal (Le et al 2010). We initialize the problem as seen in Fig. 23 and minimize the $p$-norm stress subject to a volume constraint $V^{*}$ of $50 \%$ of the design domain. We also include a compliance constraint to help the optimizer converge in this problem with highly localized stress concentrations. The compliance is constrained to be less than $150 \%$ of the compliance of an entirely filled design domain (see Fig. 23b). Our numerical experi- 


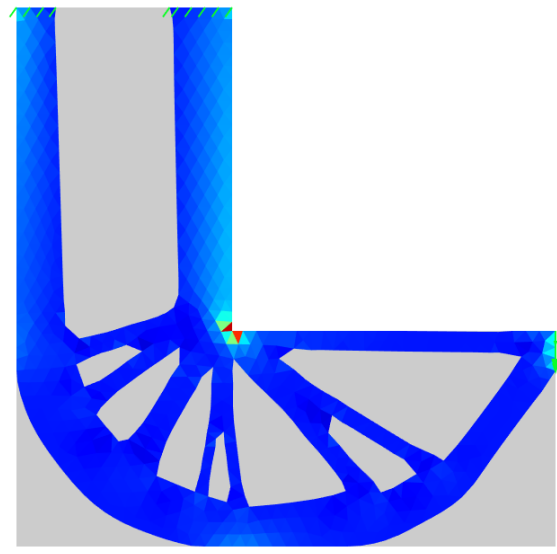

(a) Compliance

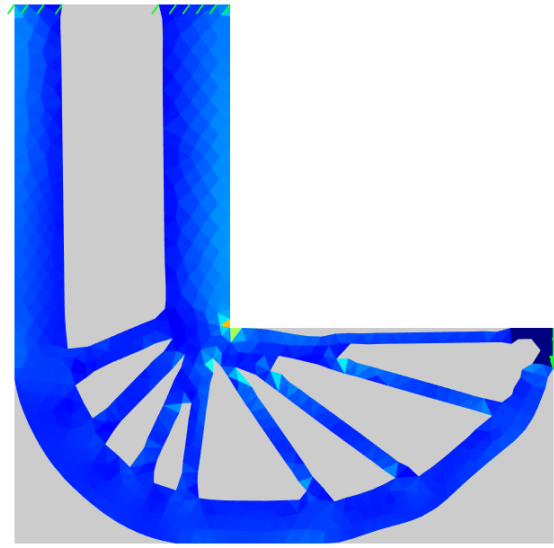

(b) $p=4$

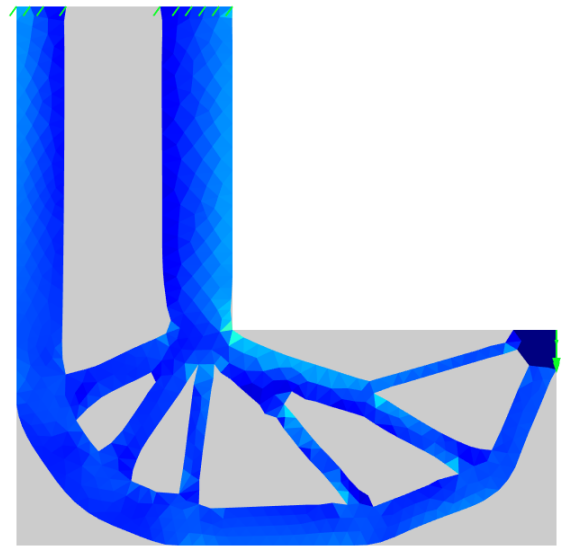

(c) $p=8$

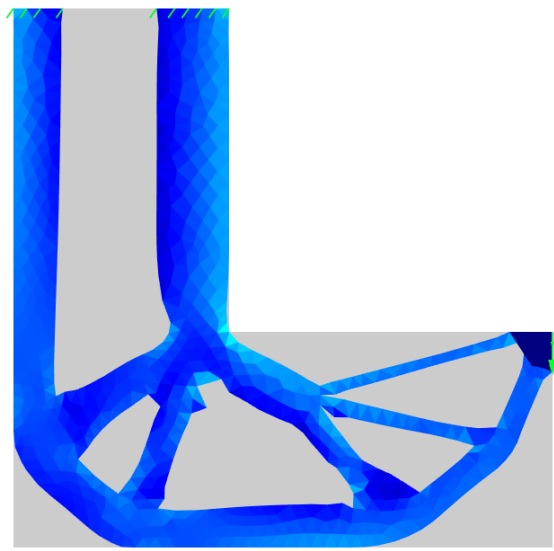

(d) $p=12$

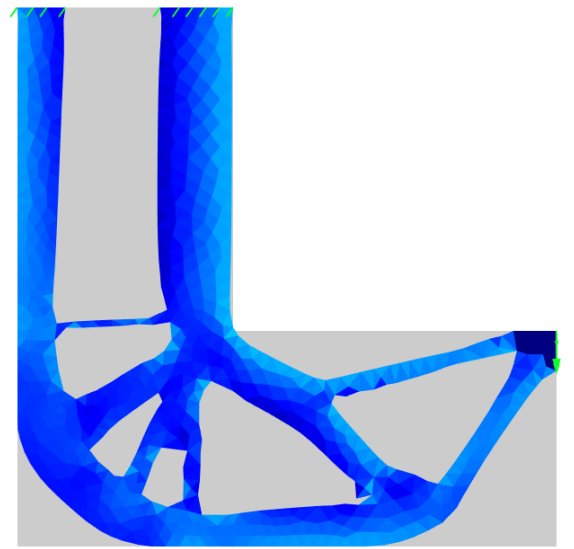

(e) $p=30$

Fig. 13 Effect of changing $p$ when minimizing the $p$-norm von Mises stress of the L-bracket beam problem for fixed $\delta_{\text {ave }}=15$. The "jet" color map represents von Mises stress. The color scale ranges from $0.00 \mathrm{~N} / \mathrm{mm}^{2}$ to $13.36 \mathrm{~N} / \mathrm{mm}^{2}$.

ments have shown that if the compliance constraint is omitted, the optimized designs for high $p$ values contain jagged boundaries away from the loads, supports and highly stressed regions. We conjecture that this is due to oscillations in the sensitivities for regions with little influence on the maximum stress; and the size of this region is relatively large for problems with high $p$ values.

Figs. 24 and 25 and Tab. 5 compare our stress minimization design to a compliance minimization design with the same volume constraint. As expected the von Mises stress is more uniformly distributed for the stressbased design.

\begin{tabular}{|c||c|c|c|}
\hline Objective & $\boldsymbol{u}^{T} \boldsymbol{K} \boldsymbol{u}$ & $\max _{i} \sigma_{i}$ & $\left(\int_{\Omega} \sigma^{30} \mathrm{~d} \Omega\right)^{\frac{1}{30}}$ \\
\hline \hline Compliance & 958168 & 17.4363 & 18.8035 \\
\hline$p$-norm stress & 1140620 & 5.785 & 6.82696 \\
\hline
\end{tabular}

Table 5 Compliance $\left(\boldsymbol{u}^{T} \boldsymbol{K} \boldsymbol{u}\right)$, maximal von Mises stress on Gauss points $\left(\max _{i} \sigma_{i}\right)$ and $p$-norm stress $\left(\left(\int_{\Omega} \sigma^{30} \mathrm{~d} \Omega\right)^{\frac{1}{30}}\right)$ for the portal design. The stress minimization is conducted with $p=30$.

\section{Discussion}

We have applied the shape and topology optimization method based on the DSC framework, proposed in Christiansen et al (2014b) for compliance minimization, to minimize the maximal von Mises stress of $2 \mathrm{D}$ structures, in the $p$-norm sense. We have shown that using higher order quadrature for the evaluation of the $p$ norm stress and second order shape functions results in smooth boundary designs without the need for ad- 


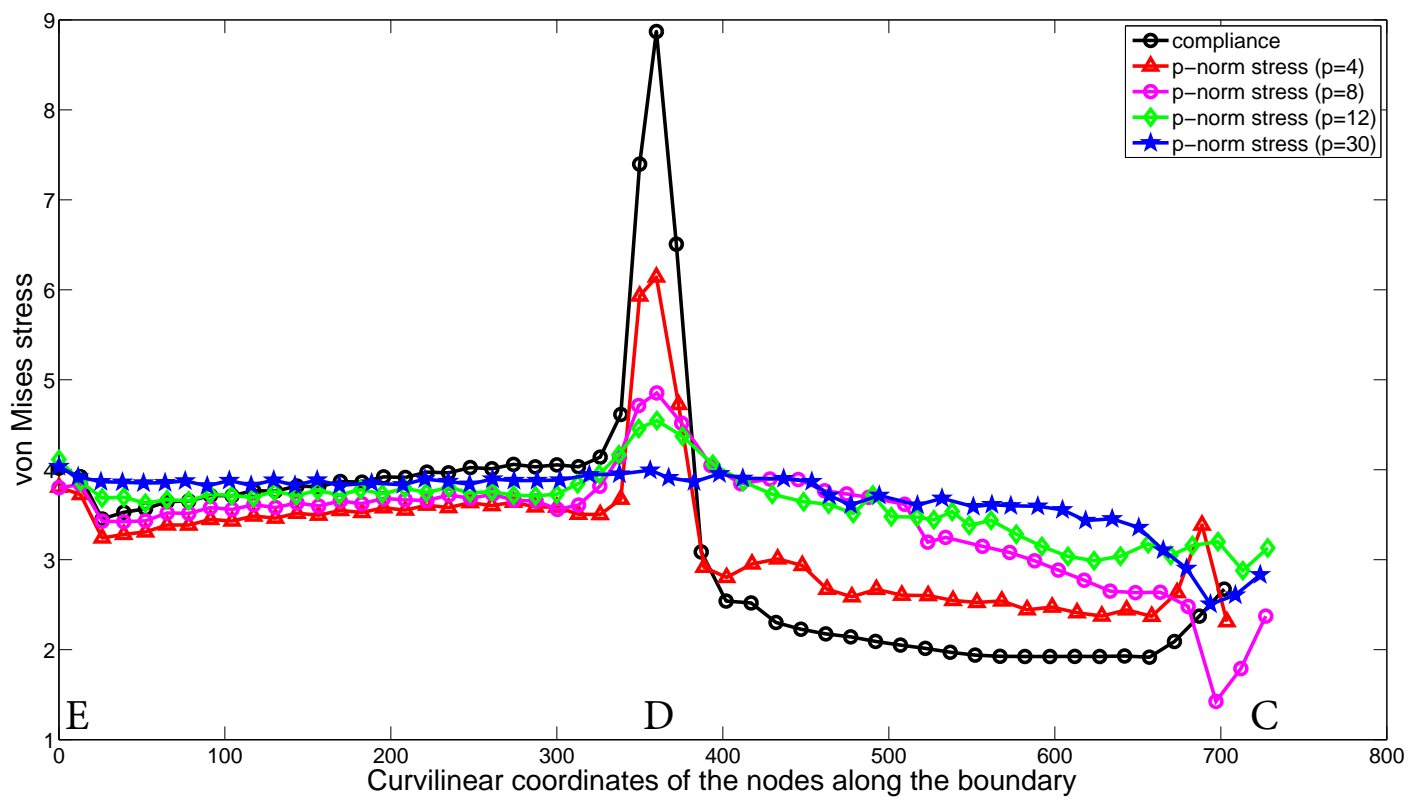

Fig. 14 Distribution of nodal von Mises stress over the inside edge that connects the support to the load, i.e. boundary curve segment EDC, for the five designs illustrated in Fig. 13 Note that the oscillations to the right are due to the loaded area which is not included in the stress norm evaluation.

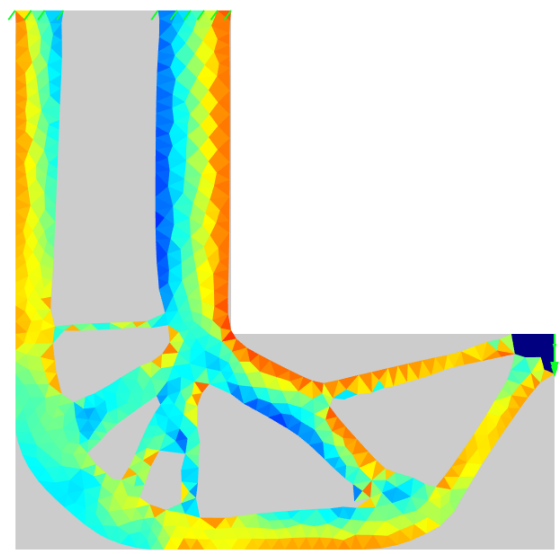

(a) $\delta_{\text {ave }}=15 \mathrm{~mm}$

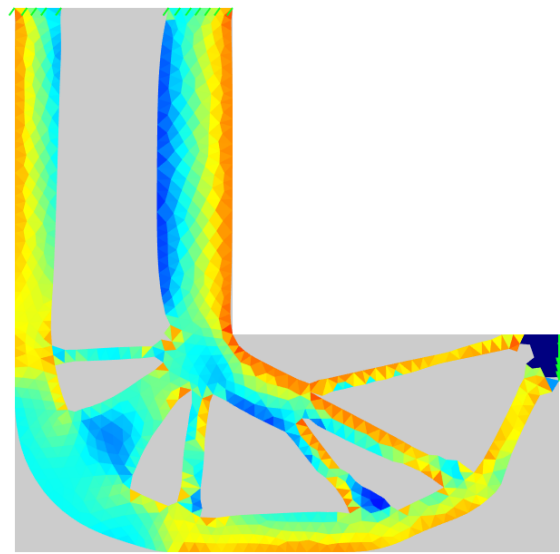

(b) $\delta_{\text {ave }}=12 \mathrm{~mm}$

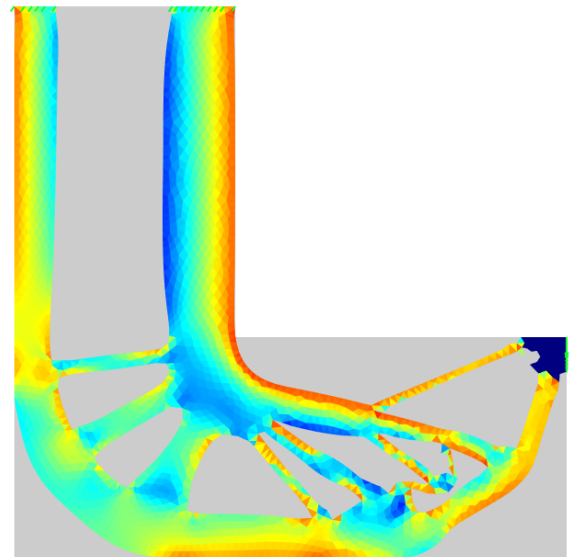

(c) $\delta_{\text {ave }}=8 \mathrm{~mm}$

Fig. 15 Effect of changing the element edge length $\delta_{\text {ave }}$ when minimizing the $p$-norm von Mises stress of the L-bracket problem with $p=30$. The "jet" color map represents von Mises stress. The color scale ranges from $0.07 \mathrm{~N} / \mathrm{mm}^{2}$ to $5.132 \mathrm{~N} / \mathrm{mm}^{2}$.

ditional smoothing. It is also demonstrated that the framework accomodates high $p$-values, e.g. $p=30$, and thus makes it possible to minimize the maximal stress. However, increasing the $p$-value causes ill-conditioning of the optimization problem and hence the scheme requires a large number of iterations. That is, the method is not very practical in its current form. However, if initial designs are obtained from classical density based topology optimization, then the costly first stage of the optimization process can be omitted, i.e. the process of reaching the final volume fraction constraint. We also remark that the omittance of the load and support regions in the evaluation of the $p$-norm stress objective increases the preprocessing task required by the designer. 


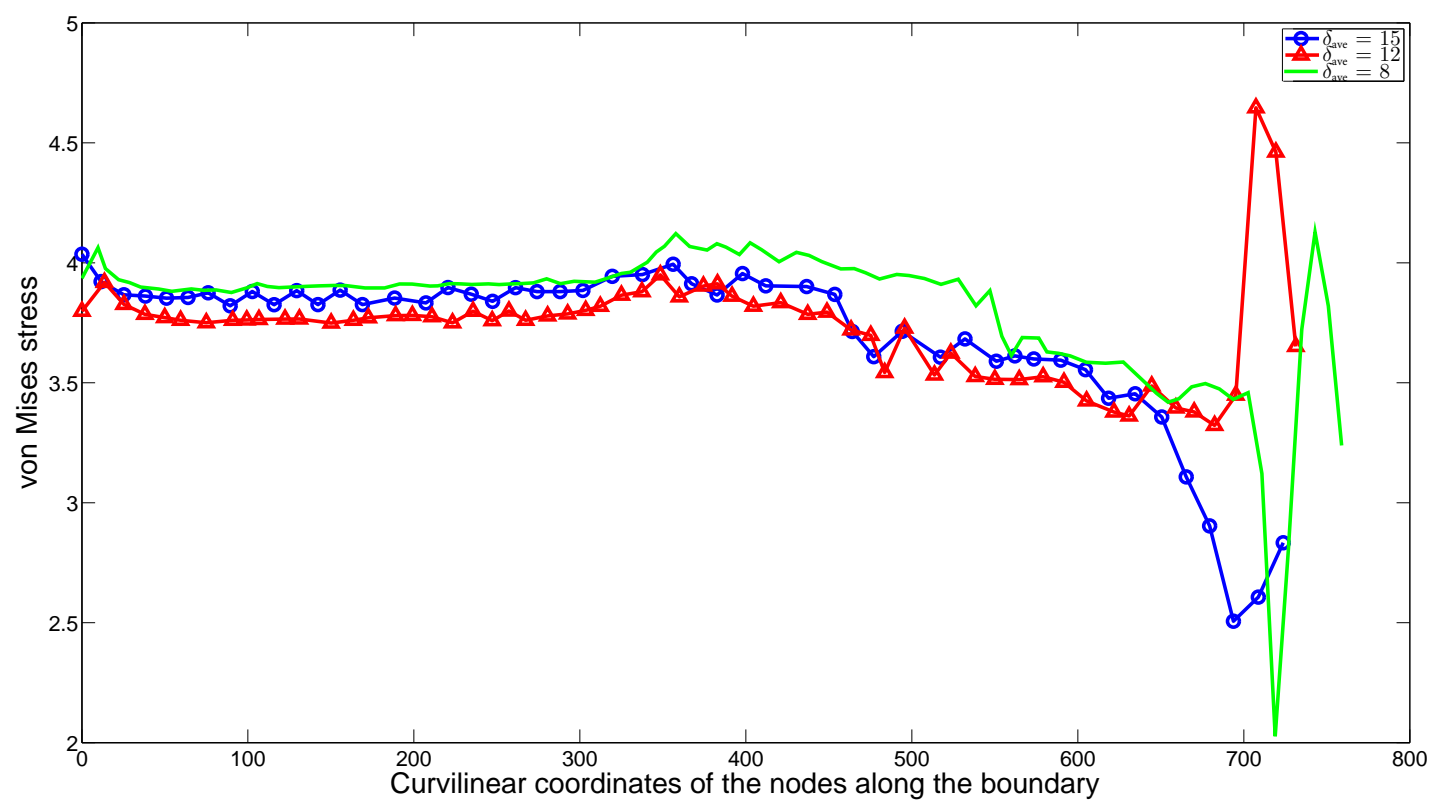

Fig. 16 Distribution of the nodal von Mises stress over the inside edge connecting the support to the load for the three designs illustrated in Fig. 15 .

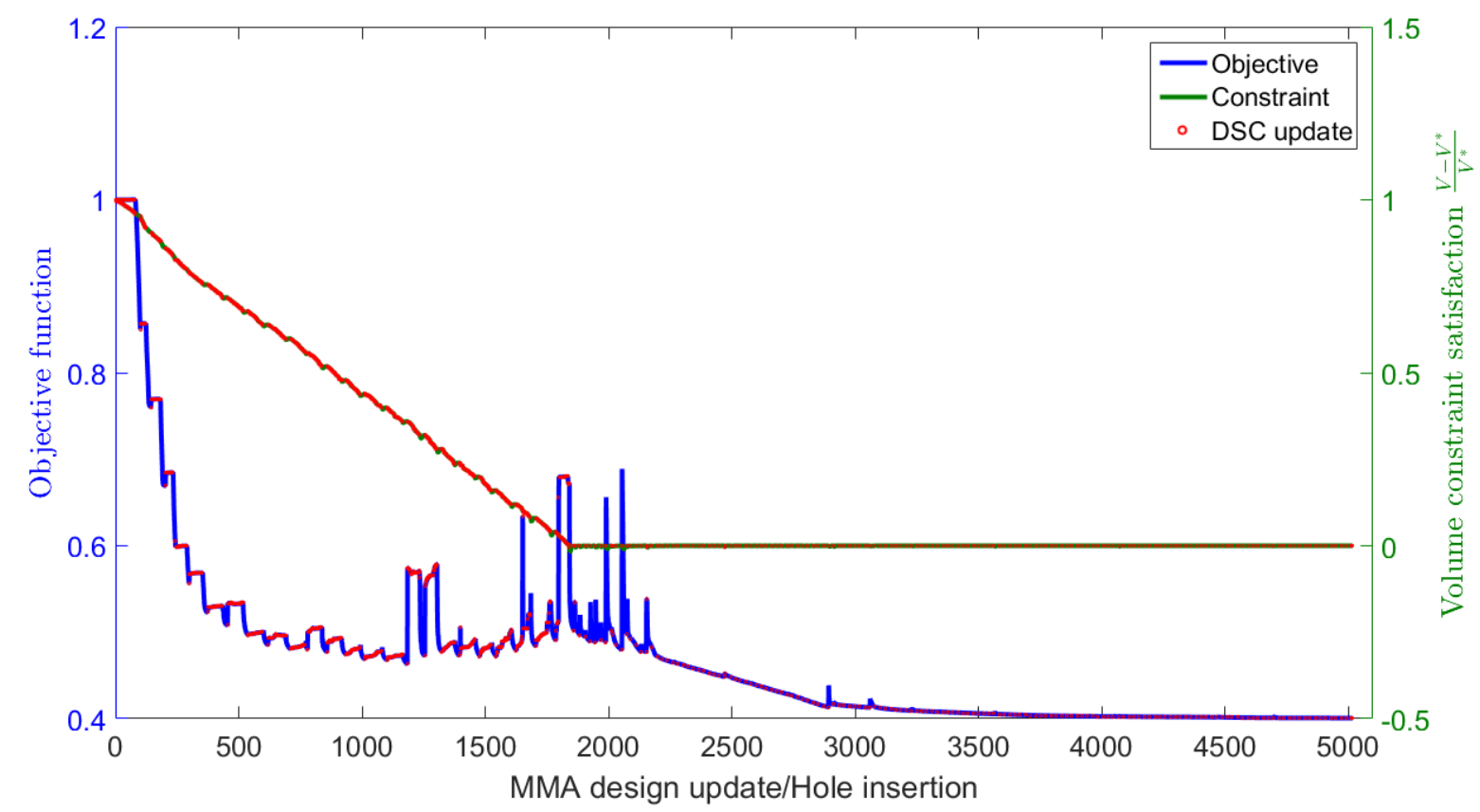

Fig. 17 The objective function and volume constraint history for $p=30, \delta_{\text {ave }}=12$. Each red point corresponds to a DSC update. 


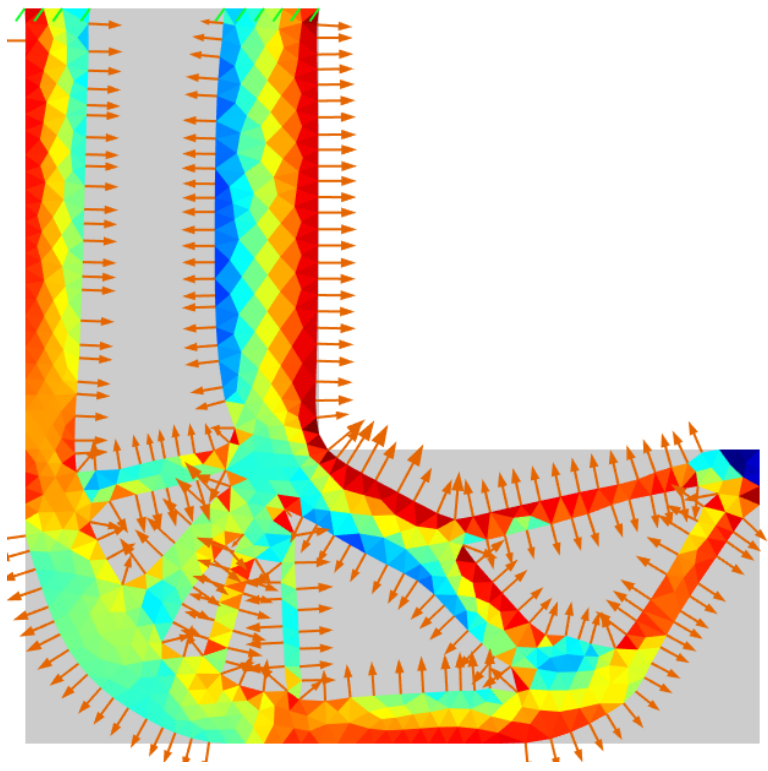

Fig. 18 Optimality condition verification (arrows corresponds to $\nabla_{i} f$ and are scaled by $\nabla_{i} f / \nabla_{i} g$ ) and stress distribution. The color scale ranges from $0.00 \mathrm{~N} / \mathrm{mm}^{2}$ to $4.24 \mathrm{~N} / \mathrm{mm}^{2}$.

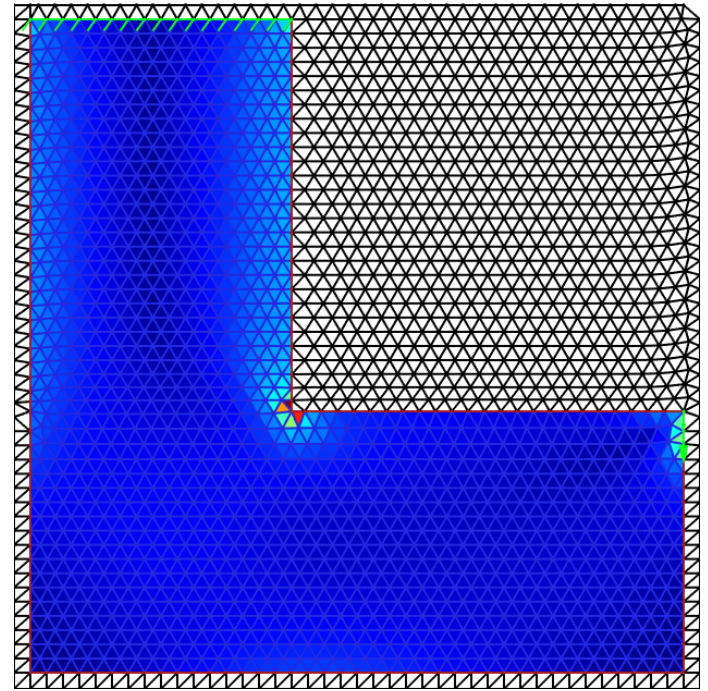

(a)

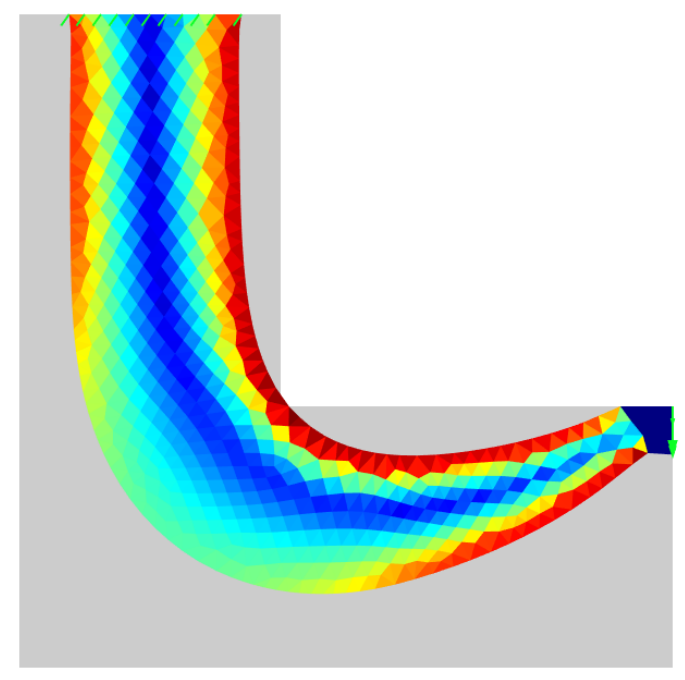

(b)

Fig. 19 The initial discretization of the L-bracket (a) without holes and the p-norm stress optimized result (b). The "jet" color map represents the von Mises stress. The grey color indicates the void region. The color scales for the initial and optimized structures range from $0.02 \mathrm{~N} / \mathrm{mm}^{2}$ to $10.84 \mathrm{~N} / \mathrm{mm}^{2}$ and $0.00 \mathrm{~N} / \mathrm{mm}^{2}$ to $8.04 \mathrm{~N} / \mathrm{mm}^{2}$, respectively.

Future work includes extending the approach to 3D - which has already been used to generate minimal compliance designs (Christiansen et al 2014a) - and improving its efficiency. However, before proceeding to a 3D implementation, the efficiency of the method must be improved. This could for example be done by the two-step approach described above, by starting with a design that fulfills the volume constraint a priori or by a continuation method in the $p$-value such that it is slowly increased from e.g. 4 to 30 . It would also be interesting to study the size of the omitted loaded and supported regions and their influence on the optimized topologies. 


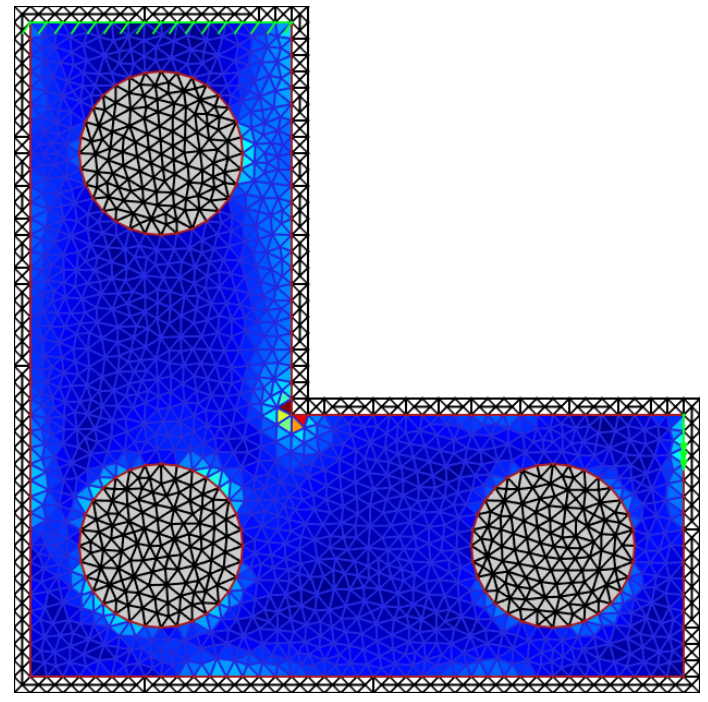

(a)

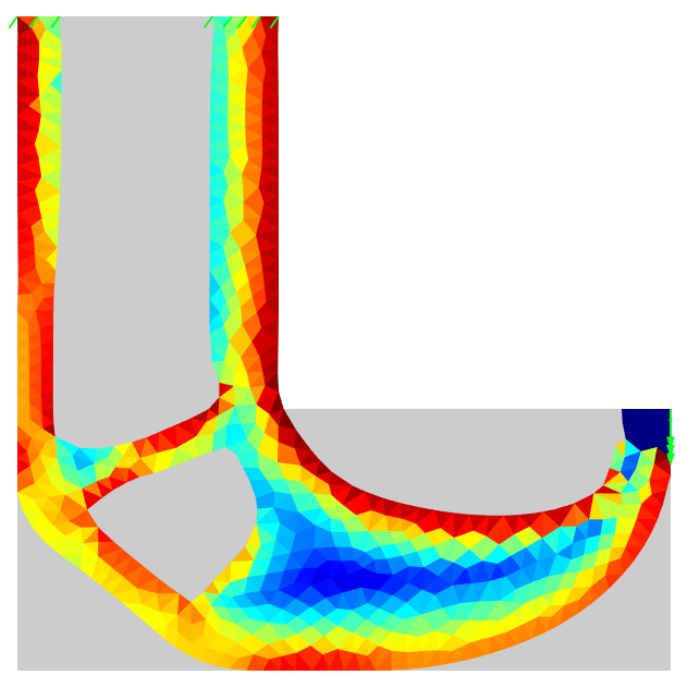

(b)

Fig. 20 The initial discretization of the L-bracket (a) with 3 holes and the $p$-norm stress optimized result (b). The "jet" color map represents the von Mises stress. The grey color indicates the void region. The color scales for the initial and optimized structures range from $0.02 \mathrm{~N} / \mathrm{mm}^{2}$ to $15.15 \mathrm{~N} / \mathrm{mm}^{2}$ and $0.00 \mathrm{~N} / \mathrm{mm}^{2}$ to $4.63 \mathrm{~N} / \mathrm{mm}^{2}$, respectively.

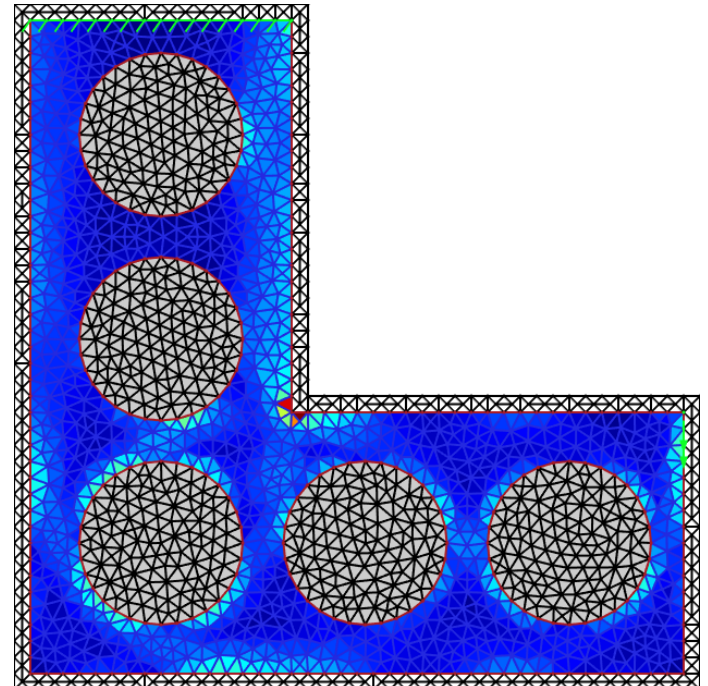

(a)

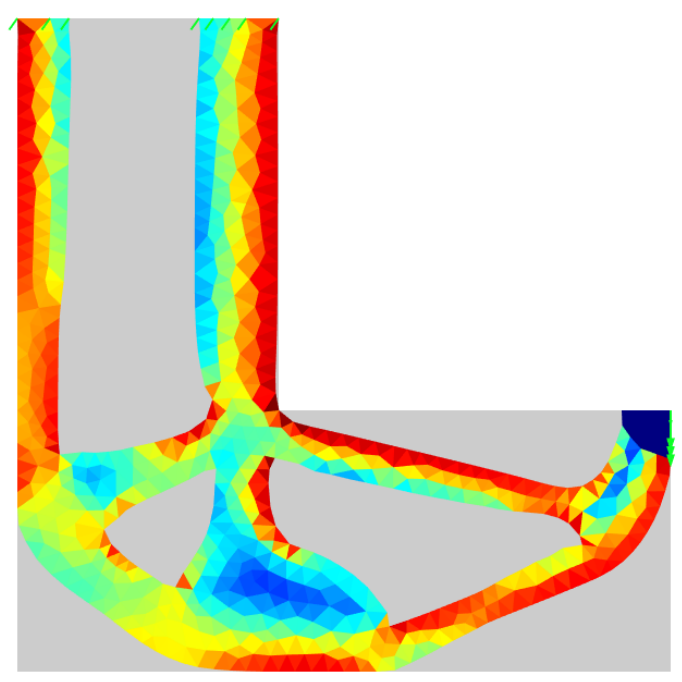

(b)

Fig. 21 The initial discretization of the L-bracket (a) with 5 holes and the p-norm stress optimized result (b). The "jet" color map represents the von Mises stress. The grey color indicates the void region. The color scales for the initial and optimized structures range from $0.02 \mathrm{~N} / \mathrm{mm}^{2}$ to $14.11 \mathrm{~N} / \mathrm{mm}^{2}$ and $0.00 \mathrm{~N} / \mathrm{mm}^{2}$ to $4.40 \mathrm{~N} / \mathrm{mm}^{2}$, respectively.

\section{Acknowledgements}

The authors appreciate the support of the Villum foundation through the grant "NextTop" as well as the EUproject "LaScISO". Also, we would like to express our gratitude to Andreas Bærentzen and Morten NobelJørgensen for assistance, support and valuable discussions. 


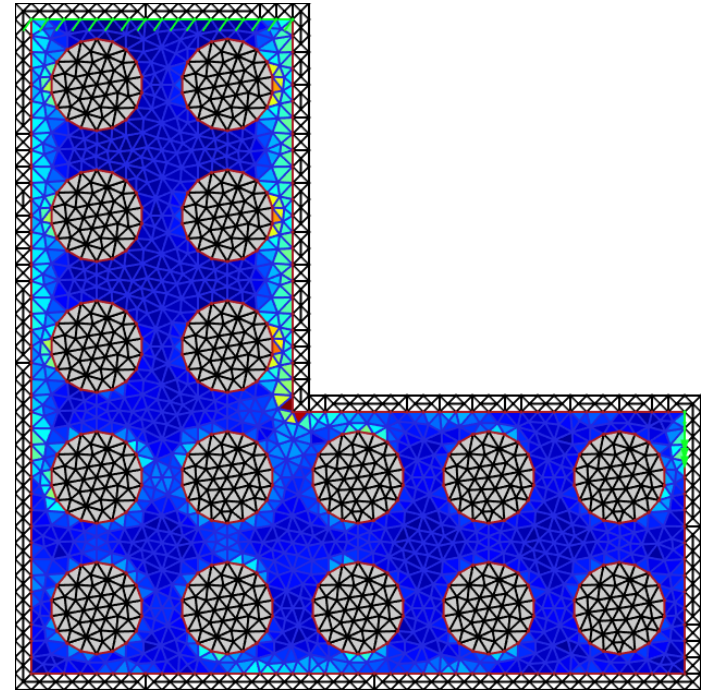

(a)

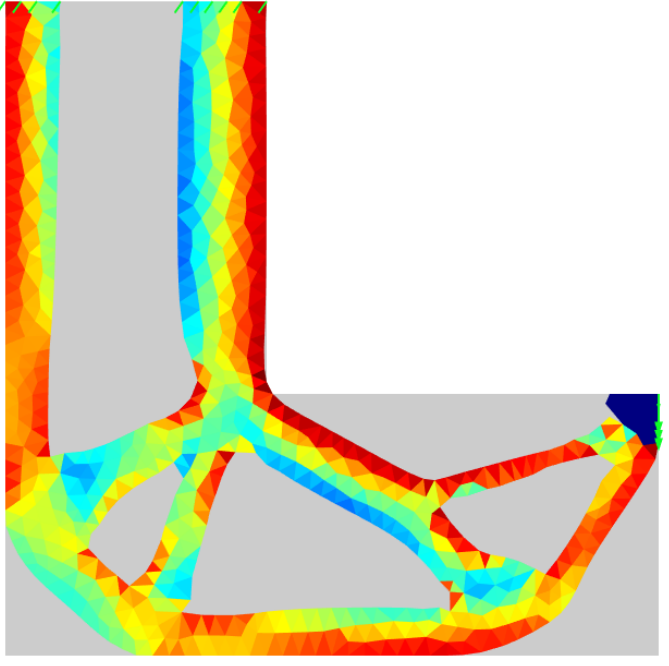

(b)

Fig. 22 The initial discretization of the L-bracket (a) with 16 holes and the p-norm stress optimized result (b). The "jet" color map represents the von Mises stress. The grey color indicates the void region. The color scales for the initial and optimized structures range from $0.02 \mathrm{~N} / \mathrm{mm}^{2}$ to $17.18 \mathrm{~N} / \mathrm{mm}^{2}$ and $0.00 \mathrm{~N} / \mathrm{mm}^{2}$ to $4.24 \mathrm{~N} / \mathrm{mm}^{2}$, respectively.

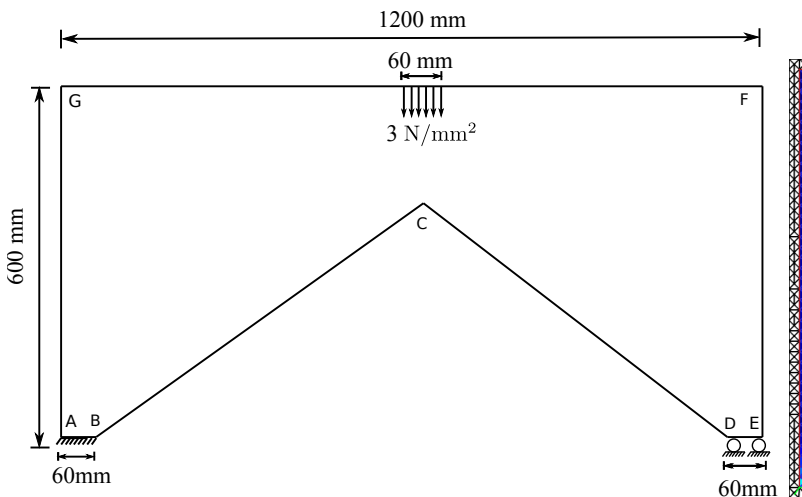

(a)

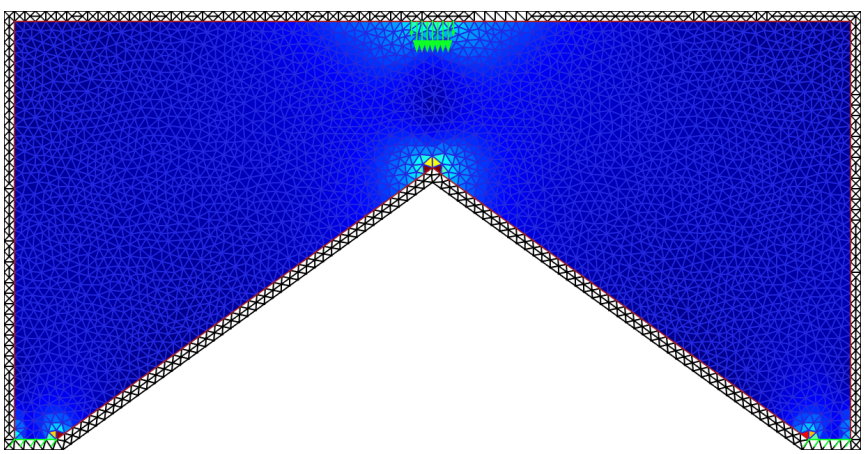

(b)

Fig. 23 Portal problem definition (a) and the initial discretization (b). The portal thickness is 1 mm and the average edge length is $\delta_{\text {ave }}=20 \mathrm{~mm}$. The "jet" color map represents the von Mises stress. The grey color indicates the void region. The color scale ranges from $0.01 \mathrm{~N} / \mathrm{mm}^{2}$ to $14.83 \mathrm{~N} / \mathrm{mm}^{2}$. 


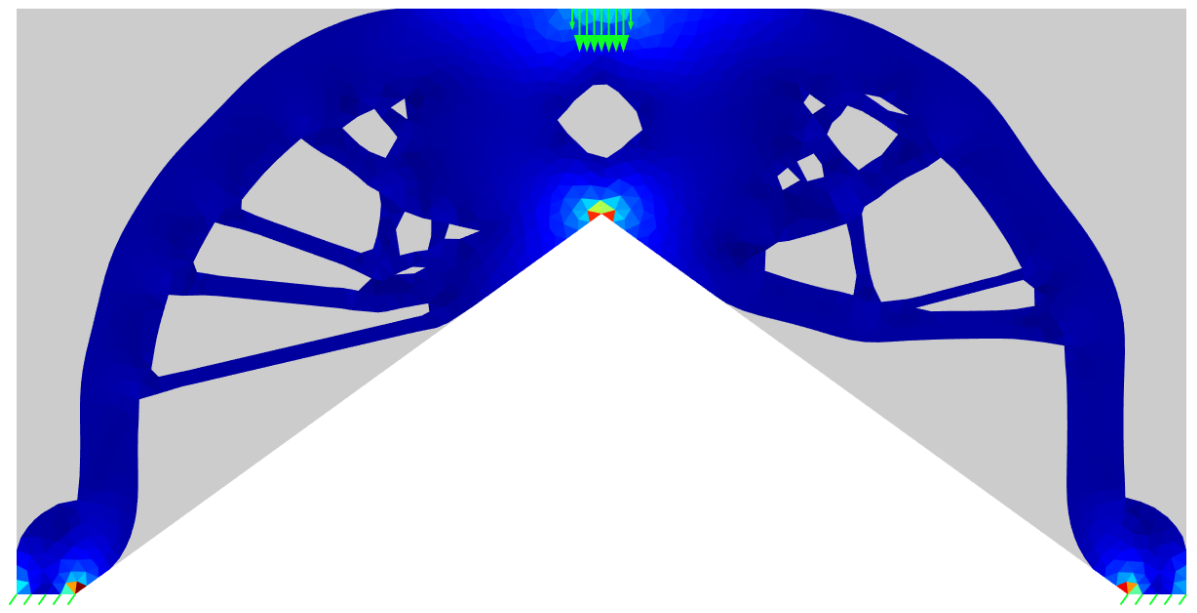

Fig. 24 Compliance minimization result of the portal problem. The "jet" color map represents the von Mises stress. The grey color indicates the void region. The color scale ranges from $0.00 \mathrm{~N} / \mathrm{mm}^{2}$ to $17.44 \mathrm{~N} / \mathrm{mm}^{2}$.

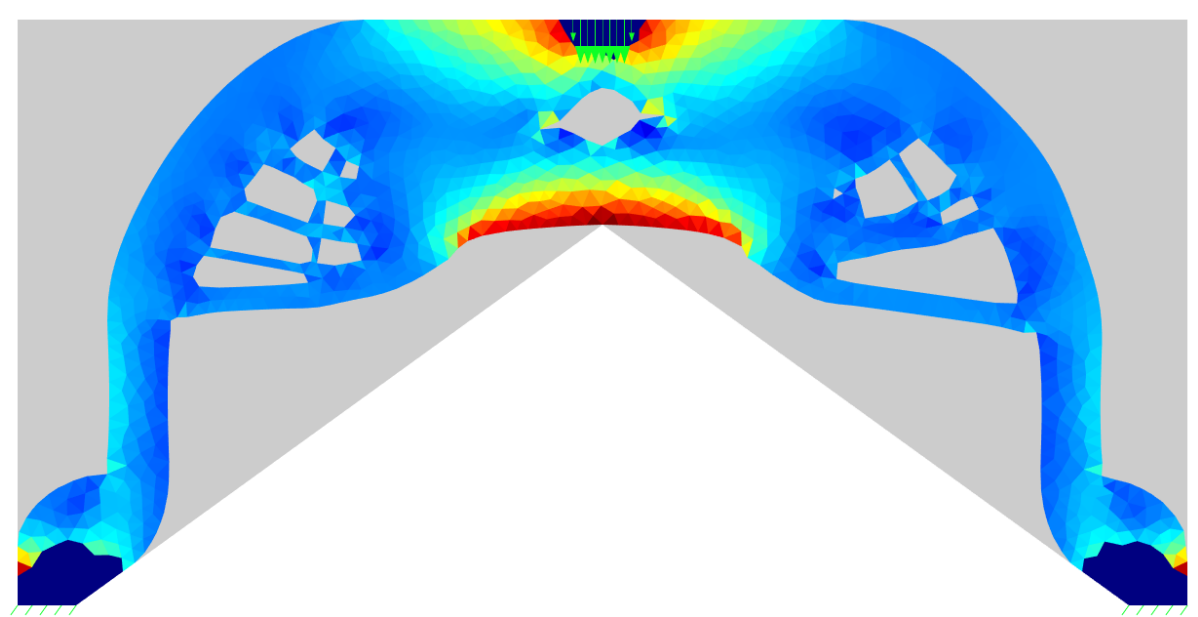

Fig. 25 Maximal stress minimization result of the portal problem. The "jet" color map represents the von Mises stress. The grey color indicates the void region. The color scale ranges from $0.00 \mathrm{~N} / \mathrm{mm}^{2}$ to $5.79 \mathrm{~N} / \mathrm{mm}^{2}$. 


\section{References}

Allaire G, Jouve F (2008) Minimum stress optimal design with the level set method. Engineering Analysis with Boundary Elements 32(11):909-918

Allaire G, Jouve F, Toader AM (2004) Structural optimization using sensitivity analysis and a level-set method. Journal of Computational Physics 194(1):363-393

Allaire G, Dapogny C, Frey P (2011) Topology and geometry optimization of elastic structures by exact deformation of simplicial mesh. Comptes Rendus Mathematique 349(17):999-1003

Allaire G, Dapogny C, Frey P (2013) A mesh evolution algorithm based on the level set method for geometry and topology optimization. Structural and Multidisciplinary Optimization 48(4):711-715

Allaire G, Dapogny C, Frey P (2014) Shape optimization with a level set based mesh evolution method. Computer Methods in Applied Mechanics and Engineering 282:22-53

Amstutz S, Novotny AA (2010) Topological optimization of structures subject to Von Mises stress constraints. Structural and Multidisciplinary Optimization 41(3):407-420

Bendsøe MP (1989) Optimal shape design as a material distribution problem. Structural Optimization 1(4):193-202

Bendsøe MP, Sigmund O (2003) Topology Optimization Theory, Methods, and Applications, second edition edn. Springer Verlag, Berlin

Bletzinger KU (2014) A consistent frame for sensitivity filtering and the vertex assigned morphing of optimal shape. Structural and Multidisciplinary Optimization 49(6):873895

Bruggi M, Venini P (2008) A mixed FEM approach to stressconstrained topology optimization. International Journal for Numerical Methods in Engineering 73(12):1693-1714

Changwen X, Minghua Y (1990) Shape optimization of structures to minimize stress concentration. Computers \& Structures 36(3):491 - 497

Cheng G, Jiang Z (1992) Study on topology optimization with stress constraints. Engineering Optimization 20(2):129148

Cherkaev AV, Grabovsky Y, Movchan AB, Serkov SK (1998) The cavity of the optimal shape under the shear stresses. International Journal of Solids and Structures 35(33):4391-4410

Christiansen AN, Bærentzen JA, Nobel-Jørgensen M, Aage N, Sigmund O (2014a) Combined shape and topology optimization of 3D structures. Computers \& Graphics (0):-, Shape Modeling International 2014

Christiansen AN, Nobel-Jørgensen M, Aage N, Sigmund O, Bærentzen JA (2014b) Topology optimization using an explicit interface representation. Structural and Multidisciplinary Optimization 49(3):387-399

Duysinx P, Bendsøe MP (1998) Topology optimization of continuum structures with local stress constraints. International Journal for Numerical Methods in Engineering 43(8):1453-1478

Duysinx P, Sigmund O (1998) New developments in handling optimal stress constraints in optimal material distributions. In: Conference Proceedings of 7th Symposium in Multidisciplinary Analysis and Optimization, AIAA/USAF/NASA/ISSMO, St. Louis Missouri, USA, vol 3, pp 1501-1509

Francavilla A, Ramakrishnan CV, Zienkiewicz OC (1975) Optimization of shape to minimize stress concentration. The Journal of Strain Analysis for Engineering Design 10(2):63-70
Ha SH, Cho S (2008) Level set based topological shape optimization of geometrically nonlinear structures using unstructured mesh. Computers \& Structures 86(13):14471455

Haftka RT, Gürdal Z (1992) Elements of Structural Optimization, 3rd edn. Kluwer Academic Publishers, Boston

Le C, Norato J, Bruns T, Ha C, Tortorelli D (2010) Stressbased topology optimization for continua. Structural and Multidisciplinary Optimization 41(4):605-620

Le C, Bruns T, Tortorelli D (2011) A gradient-based, parameter-free approach to shape optimization. Computer Methods in Applied Mechanics and Engineering 200(9-12):985-996

Misztal MK, Bærentzen JA (2012) Topology adaptive interface tracking using the deformable simplicial complex. ACM Transactions on Graphics 31(3):No. 24

Querin O, Steven G, Xie Y (1998) Evolutionary structural optimisation (ESO) using a bidirectional algorithm. Engineering Computations 15(8):1031-1048

Sanford R (2003) Principle of Fracture Mechanics. Prentice Hall

Savin G (1961) Stress concentrations around holes. New YorkPergamon Press

Sternberg E, Koiter WT (1958) The wedge under a concentrated couple: a paradox in the two-dimensional theory of elasticity. Journal of Applied Mechanics 25(575):958

Svanberg K (1987) The method of moving asymptotes - a new method for structural optimization. International Journal for Numerical Methods in Engineering 24(2):359-373

Svanberg K (2002) A class of globally convergent optimization methods based on conservative convex separable approximations. SIAM J on Optimization 12(2):555-573

Svanberg K, Werme M (2007) Sequential integer programming methods for stress constrained topology optimization. Structural and Multidisciplinary Optimization 34(4):277-299

Wang M, Wang X, Guo D (2003) A level set method for structural topology optimization. Computer Methods in Applied Mechanics and Engineering 192(1):227-246

Yamasaki S, Nomura T, Kawamoto A, Sato K, Nishiwaki S (2011) A level set-based topology optimization method targeting metallicwaveguide design problems. International Journal for Numerical Methods in Engineering 87(February):844-868 